\title{
Two-Dimensional Electron Systems in Magnetic Fields: The Current Equipartition Law
}

\author{
Tsuyoshi Ueta \\ Physics Laboratory, The Jikei University School of Medicine, 8-3-1 Kokuryo-cho, Chofu, Tokyo182-8570, Japan \\ Correspondence should be addressed to Tsuyoshi Ueta, tsuyoshi_ueta2@ybb.ne.jp \\ Received 15 March 2011; Revised 9 July 2011; Accepted 30 August 2011 \\ Academic Editor: Sergio E. Ulloa \\ Copyright () 2011 Tsuyoshi Ueta. This is an open access article distributed under the Creative Commons Attribution License, \\ which permits unrestricted use, distribution, and reproduction in any medium, provided the original work is properly cited.
}

We consider two-dimensional randomly deformed circular quantum dots with two attached waveguides (an emitter and a collector) in magnetic fields as an electronic analogy of the blackbody radiation. Transport properties through them are numerically investigated. The fraction of the current carried by each propagating mode in the collector is computed for transmission currents when each propagating mode is incident. By taking the statistical average in shape, it is shown that a universal frequency distribution is obtained for a sufficiently deformed system even though magnetic fields are so strong that electron waves form edge states. Then, the transmission currents are randomly distributed over all propagating modes. On average, each propagating mode carries the same current as in the absence of a magnetic field. It is also confirmed that a finite size dot cannot be a model of a reservoir even if it is chaotic.

\section{Introduction}

In the present paper, we consider electron transport in twodimensional (2D) electron systems. Especially, we attempt to investigate a reservoir, that is, an electron source in quantum theory. In theoretical and numerical treatment of 2D electron transport, a billiard ball model is useful and has often been applied. In a billiard ball model, reservoirs are taken into consideration, and a reservoir is modeled by an infinitely large cavity. For electrons in the reservoir, uniform spatial distribution and random motion are assumed. When electrons go into a waveguide from such a reservoir, the angular distribution, namely, the probability of incidence at an angle of $\theta$ is given by $P(\theta)=(1 / 2) \cos \theta[1]$.

In quantum theory, on the other hand, usually reservoirs are basically left out of consideration. When multiple modes propagate in a waveguide, a wavefunction in the waveguide is expressed by a superposition of eigenfunctions of the propagating modes. The coefficients for the superposition are usually assumed to have the same phase and the same amplitude. Actually, the properties of the coefficients are determined by the reservoir, so that we need to consider a reservoir also in quantum theory. This problem was pointed out and discussed by Kawamura et al. [2] for the first time. In the reference, however, no solution was presented and it had been an open question.

Several researchers consider that a reservoir does not need to be taken into consideration even in quantummechanics since quantum mechanical description of transport problems has been very successful. Surely, so long as we deal with transport properties of conductors, such as calculation of conductance, it is true. However, the reservoir constitutes a part of the physical system under consideration, and we cannot avoid dealing with it quantum-mechanically. When we calculate a wave function in a conductor, especially like a diffraction pattern, the details of a reservoir is indispensable. Actually, in many cases, in order to calculate the wavefunction in a system, the eigenfunctions in a lead are superposed with the same phase and the same amplitude [3]. However, it must be said that this is without foundation.

It is impossible to treat an infinite system like a classical model of a reservoir, so I proposed a finite size model of a reservoir. In the author's previous work $[4,5]$, the transport properties of a random-shaped circular quantum dot connected to two waveguides have been analyzed quantum mechanically and classically. Actually, the analysis of eigenstates and transport properties of an electron wave 


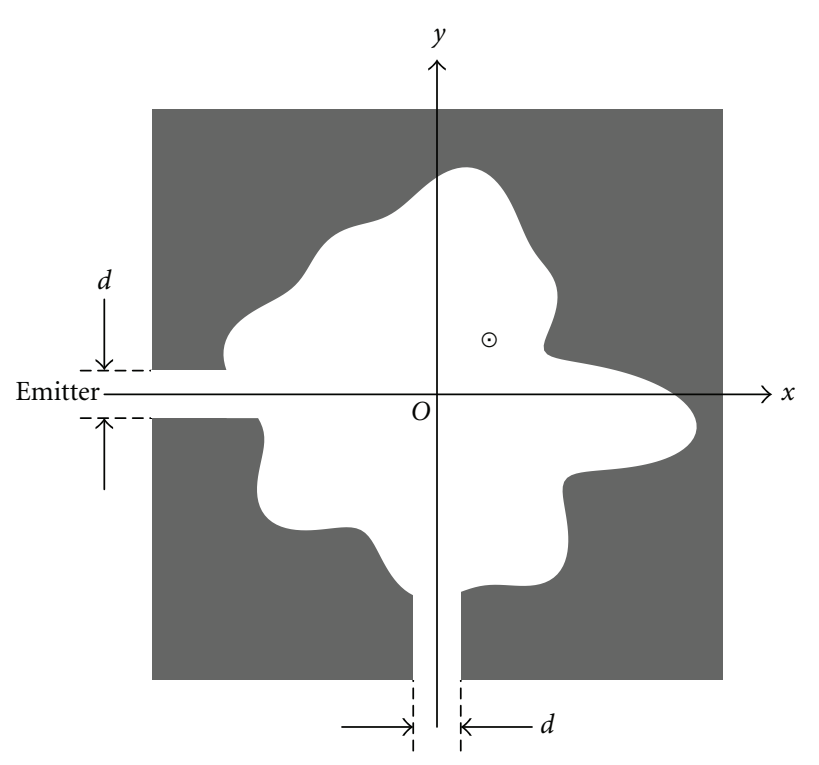

Figure 1: An example of a random-shaped quantum dot with two waveguides and the definition of the coordinate system.

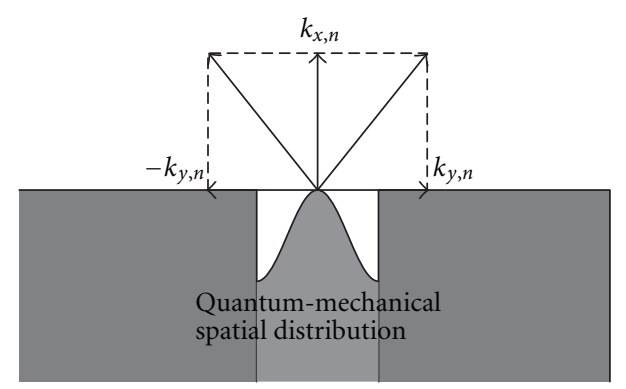

FIGURE 2: Schematic description of the quantized billiard model. The quantization of momentum of electrons and the quantummechanical probability distribution within the emitter are assumed.

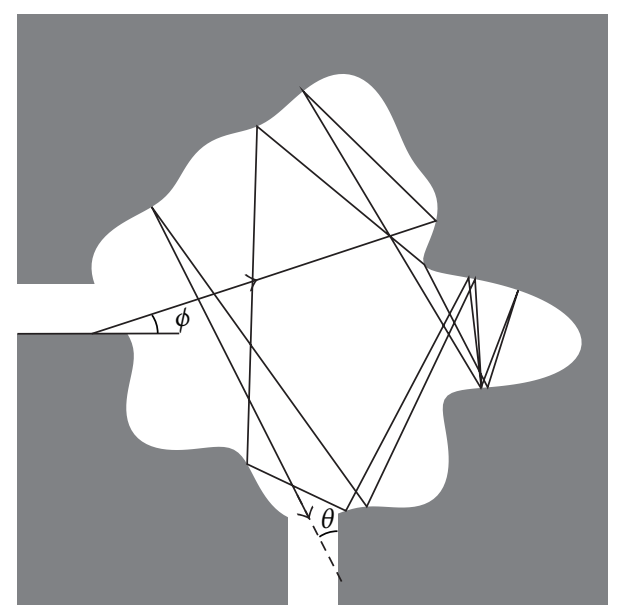

FIgURE 3: A sample trajectory from the emitter to the collector.

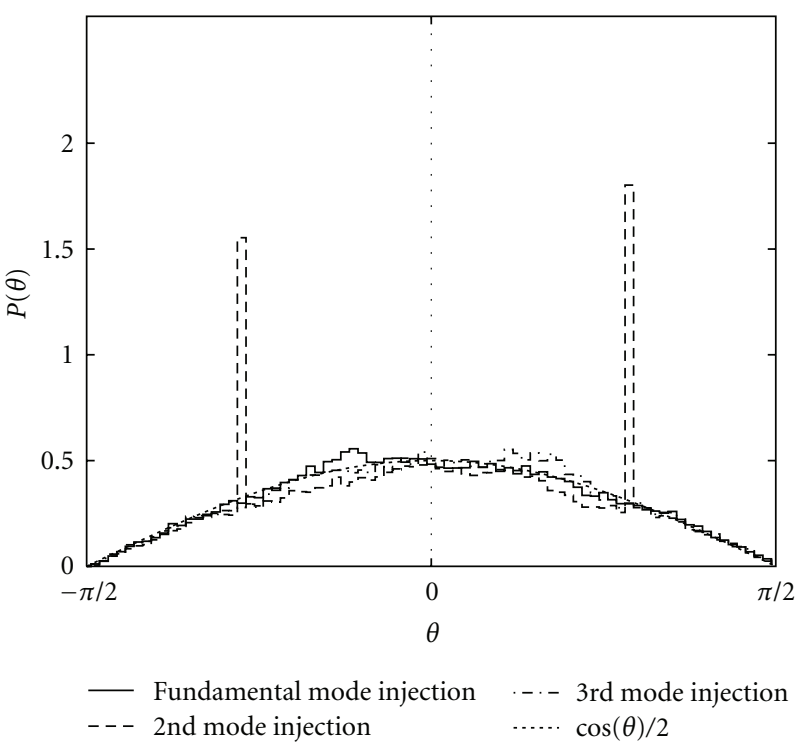

Figure 4: Angular distributions $P(\theta)$ of the electrons transmitted to the collector for the injection of each three propagating modes. $K d=10, r_{\max }=4, a_{\max }=1.5$.

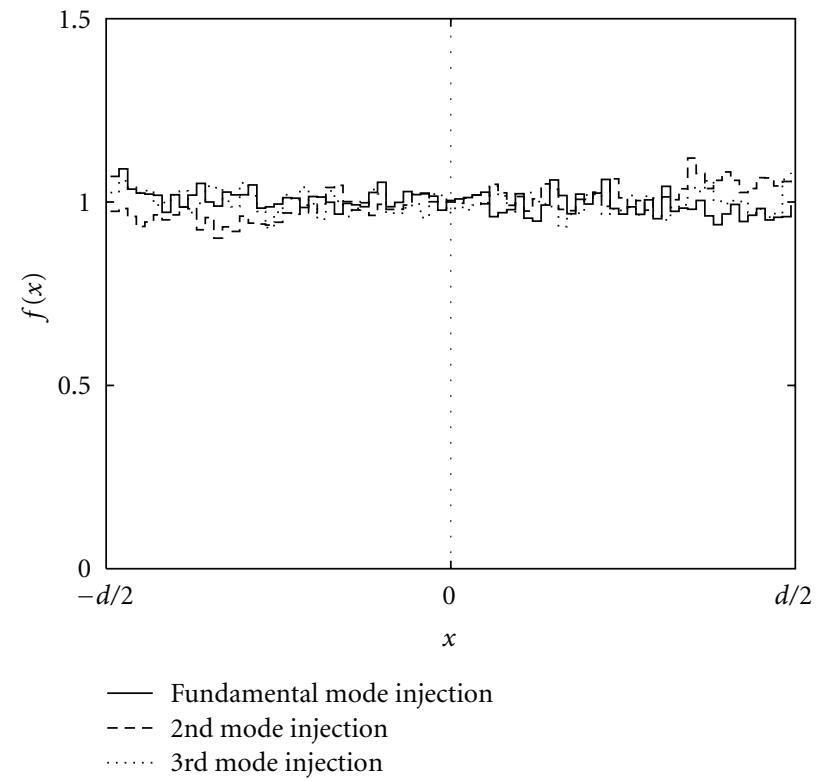

Figure 5: Spatial distributions $f(x)$ of the transmitted electrons for the incidence of each three propagating modes.

within a deformed circular quantum dot has been studied from a viewpoint of quantum chaos [6-8].

By taking the statistical average with respect to shape, the effect of the degree of randomness of shape on the transport properties has been studied $[4,5]$. The fraction of the current carried by each propagating mode in the waveguide has been derived for transmission and reflection currents when each propagating mode is incident. It has been shown that a universal frequency distribution

$$
\begin{aligned}
f(p) & =(N-1)(1-p)^{N-2}, \\
N & =\text { number of propagating modes, }
\end{aligned}
$$




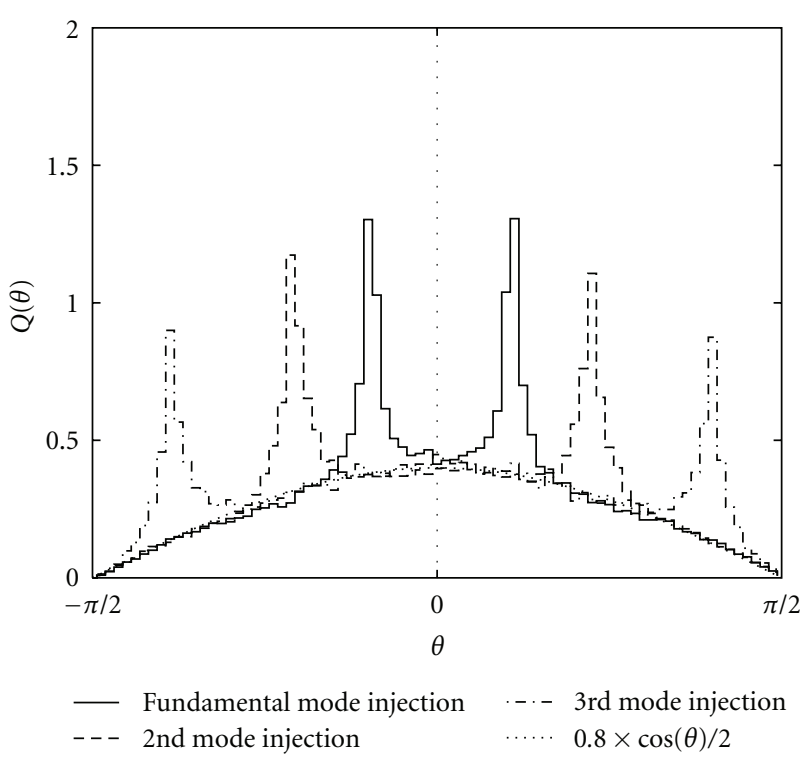

Figure 6: Angular distributions $Q(\theta)$ of the reflected electrons for the injection of each three propagating modes; $K d=10, r_{\max }=4$, $a_{\max }=1.5$.

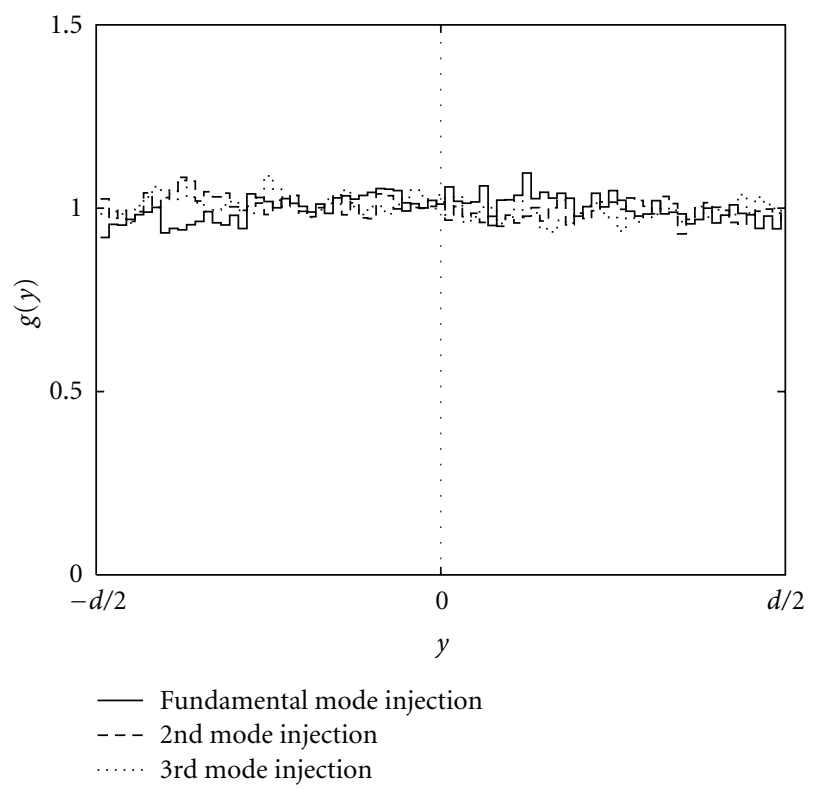

FIGURE 7: Spatial distributions $g(y)$ of the reflected electrons for the injection of each three propagating modes.

is obtained if the randomness of the shape exceeds a certain value. In a sufficiently random system, the transmission currents are randomly distributed over all propagating modes. However, on average, each propagating mode carries the same current. This result, considered as a particle reservoir model, is compared with the characteristics of the classical reservoir model.

This result demonstrates universal conductance fluctuation (UCF) by a concrete calculation from a certain point of view [9-17]. Furthermore, this result shows that for universal

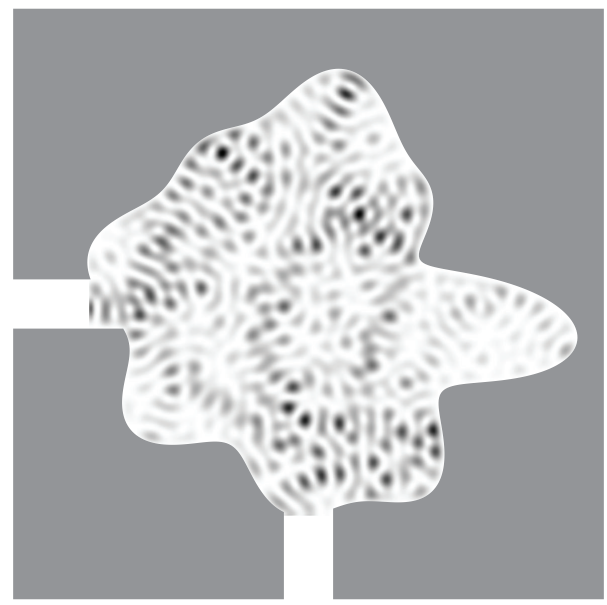

Figure 8: A contour plot of an electron density in a sample dot for $K d=\sqrt{32 \pi}, \widetilde{B}=4$, and the fundamental mode incidence.

conduction, scattering on a rough boundary is sufficient and that scatterers are not necessary within a system.

In the presence of a magnetic field, on the other hand, disturbance and dephasing of a wavefunction may not be enough, since the diffraction of an electron wave is suppressed and it forms the edge state and propagates along the boundary of the system. Therefore, it is unclear whether the universal transport properties through a quantum dot are realized.

It is often expected that an electron wave is sufficiently dephased within a chaotic quantum dot of finite size and that the transport properties through it agree with those proposed in this paper [18-27]. Even within a chaotic quantum dot at least of finite size, as shown in the last section, the universal distribution characteristics of current never appear. Thus, I emphasize here that even a chaotic quantum dot cannot become a model of a reservoir if it is finite.

\section{Reservoir Model}

We take an analogy from Planck's blackbody radiation [28]. A reservoir is defined as a cavity in which an incident wave is sufficiently dephased. It is different from blackbody radiation in that the energy of electrons is restricted to Fermi energy, since we consider a $2 \mathrm{D}$ electron system formed within a semiconductor at low temperatures. Here, we introduce statistics of random-shaped quantum dots as the mechanism of dephasing [4].

A random-shaped quantum dot is defined as a randomly deformed circular dot (Figure 1) according to [8]. The shape is expressed in the polar coordinates as

$$
r(\theta)=r_{\max }+\sum_{n=1}^{N} a_{n} \frac{a_{\max }}{2} \cos \left(n \theta+\pi \delta_{n}\right),
$$

where $r_{\max }$ and $a_{\max }$ are the radius of the original circular dot and the parameter for the degree of deformation, respectively. Both $a_{n}$ and $\delta_{n}$ are independent uniform random numbers in the range from 0 to 1 . In the present paper, we 

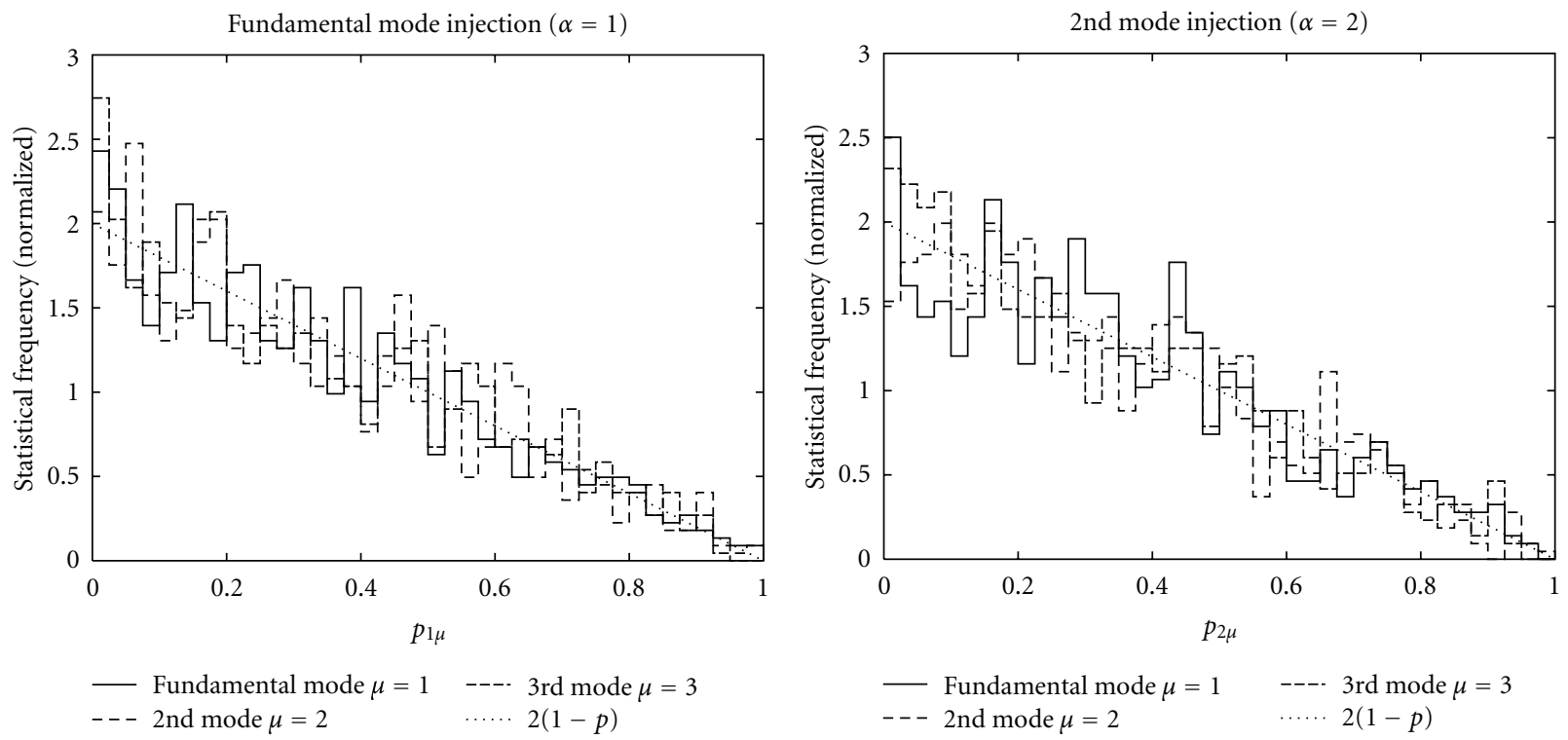

(a)

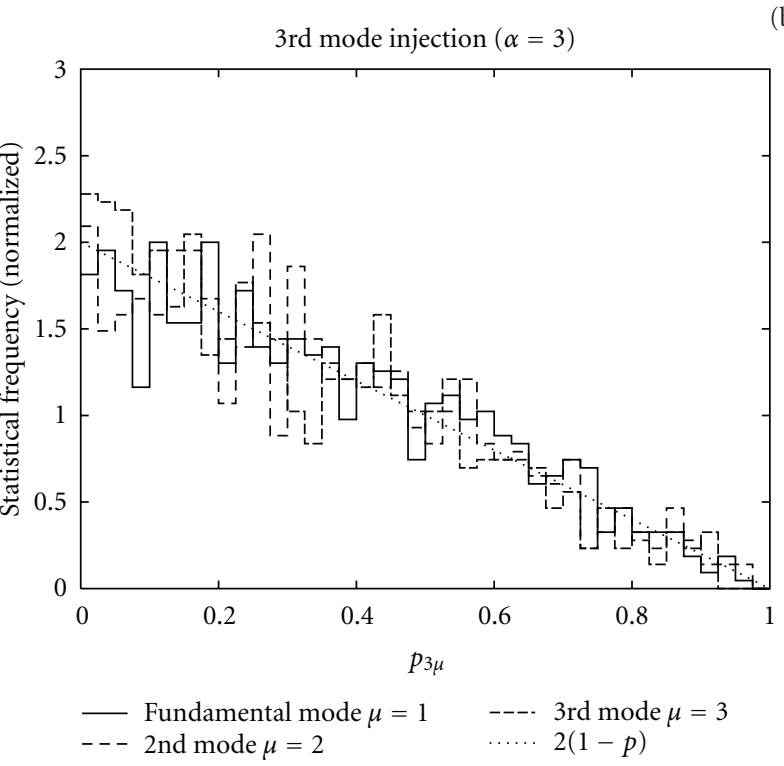

(b)

(c)

FIGURE 9: Frequency distributions for the fraction $p_{\alpha \mu}$ of each propagating mode in the transmission current carried by each propagating mode when each of three propagating modes is incident for $K d=\sqrt{32 \pi}, \widetilde{B}=4$.

set $N=9$ and $r_{\max }=4$. An emitter of width $d$ parallel to the $x$-axis and a collector with the same width are attached to the dot defined in this manner parallel to the $y$-axis. The number of samples to take statistics is 1000 .

\section{Classical Analysis}

3.1. Quantized Billiard Model. I have already reported in [5] that our model can reproduce the properties of the infinitely large classical model of a reservoir in the absence of a magnetic field. It is, however, published only Japanese, so I would like to review the results here, briefly. It is an essential and necessary condition for our model to be acceptable as a model of a reservoir. For the purpose, we employ a quantized billiard model (QBM) [29], since it makes it easy to compare the results with those of quantum theory.

In QBM, electrons in a dot are assumed to obey Newtonian equations of motion as a usual billiard model. The initial condition at the orifice of the emitter is, however, given according to quantum theory, so that quantized transverse and longitudinal momenta,

$$
\begin{gathered}
\hbar k_{y, n} \equiv \pm \hbar\left(\frac{n \pi}{d}\right), \\
\hbar k_{x, n}=\hbar \sqrt{K^{2}-k_{y, n}^{2}},
\end{gathered}
$$




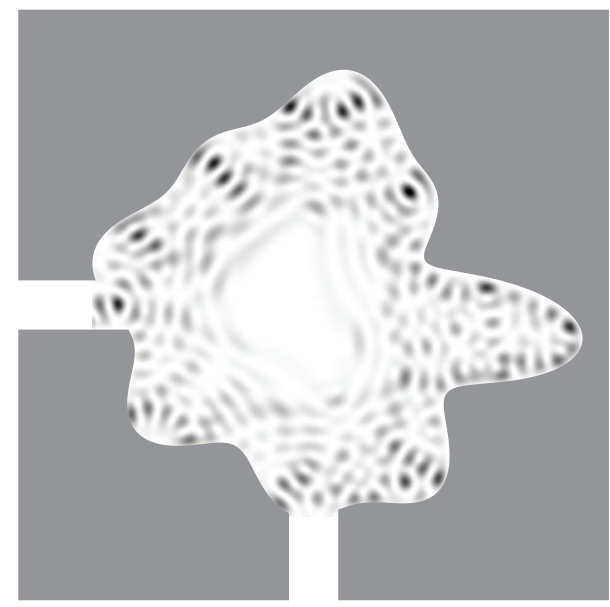

FIGURE 10: A contour plot of an electron density in a sample dot for $K d=\sqrt{32 \pi}, \widetilde{B}=9$, and the fundamental mode incidence.

are assumed when the incident wave belongs to the $n$th transverse mode. Here, $K$ is the total wavenumber of an electron, that is, the Fermi wavenumber. The spatial distribution of electrons within the emitter is given quantum mechanically as follows:

$$
\rho(y)=\frac{2}{d} \sin ^{2}\left(k_{y, n}\left(y+\frac{d}{2}\right)\right), \quad\left(-\frac{d}{2} \leq y \leq \frac{d}{2}\right) .
$$

The case of $n=1$ is illustrated in Figure 2. Figure 3 shows an example of trajectory from the emitter to the collector.

3.2. Numerical Results. The numerical simulations are performed for $K d=10$ and $a_{\max }=1.5$. In Figure 4, angular distributions of electrons in the collector are $P(\theta)$ shown for each propagating mode incidence. The angular distribution for the second mode incidence has two spikes at $\theta \sim$ $\pm 0.283 \pi$. These are attributed to electrons belonging to the second mode in the emitter that go into the collector directly without colliding with the wall of the dot.

Whether this phenomenon occurs depends on the shape of the system and on the initial position on the orifice of the emitter. In fact, the incident angle $\phi_{2}$ of electrons belonging to the second mode within the emitter is given by $\left|\phi_{2}\right|=\sin ^{-1}(2 \pi / K d) \sim 0.2163 \pi$, so that electrons with $\phi_{2} \sim-0.2163 \pi$ directly go into the collector and then the angle of incidence to the collector is $\phi_{2}+\pi / 2 \sim 0.2837 \pi$, since the collector is placed at right angles to the emitter. The angle certainly agrees with that of one spike at $\theta \sim$ $0.283 \pi$. The reason why the other spike also appears at a negative angle $\theta \sim-0.283 \pi$ is that electrons incident to the collector may collide on the right-side wall of the collector before electrons go across the counting line. For the fundamental and third mode incidence, no spike appears, since the angle of incidence is different by the incident mode. Except the spikes, the angular distribution functions for any incidence agree with that of the infinitely large classical model of a reservoir, that is, $P(\theta)=(1 / 2) \cos \theta$. The spatial distributions $f(x)$ of electrons at the orifice of the collector are plotted in Figure 5. We see that electrons are uniformly distributed within the collector for any incidence.
The angular distributions of the electrons returned to the emitter again, the so-called reflected electrons, $Q(\theta)$ are shown in Figure 6 for the same value of parameters as the case of the transmitted electrons. In this case, we see the distribution for any incident mode has two broad spikes whose positions are symmetric with respect to the origin. The angle where the twin spikes are located varies with the incident mode.

The incident angles of electrons belonging to the fundamental, second, and third mode within the emitter are

$$
\begin{aligned}
& \left|\phi_{1}\right|=\sin ^{-1}\left(\frac{\pi}{K d}\right) \sim 0.1017 \pi, \\
& \left|\phi_{2}\right|=\sin ^{-1}\left(\frac{2 \pi}{K d}\right) \sim 0.2163 \pi, \\
& \left|\phi_{3}\right|=\sin ^{-1}\left(\frac{3 \pi}{K d}\right) \sim 0.3915 \pi,
\end{aligned}
$$

respectively. These agree with the angles where the twin spikes occur for respective incident modes in Figure 6. This fact implies that these spikes are caused by reentrant of the emitted electrons to the emitter after the first and almost perpendicular collision with the wall of the dot. Each angular distribution except the twin spikes shows remarkable agreement with $0.8 \times(1 / 2) \cos \theta$. Hence, we see that about $10 \%$ of the reflected electrons form one of the twin spikes for any incident mode. The remainder, namely, $80 \%$ of the reflected electrons, are diffusive and sufficiently disturbed. The spatial distributions $g(y)$ of the reflected electrons are plotted in Figure 7 for incidence of each propagating mode. They are also uniformly distributed within the emitter for any incident mode. Therefore, our random-shaped dot model is acceptable as a model of a reservoir, at least, classically and in the absence of a magnetic field.

\section{Quantum-Mechanical Analysis in Magnetic Fields}

In the present study, we consider the case in the presence of a uniform magnetic field quantum mechanically. 


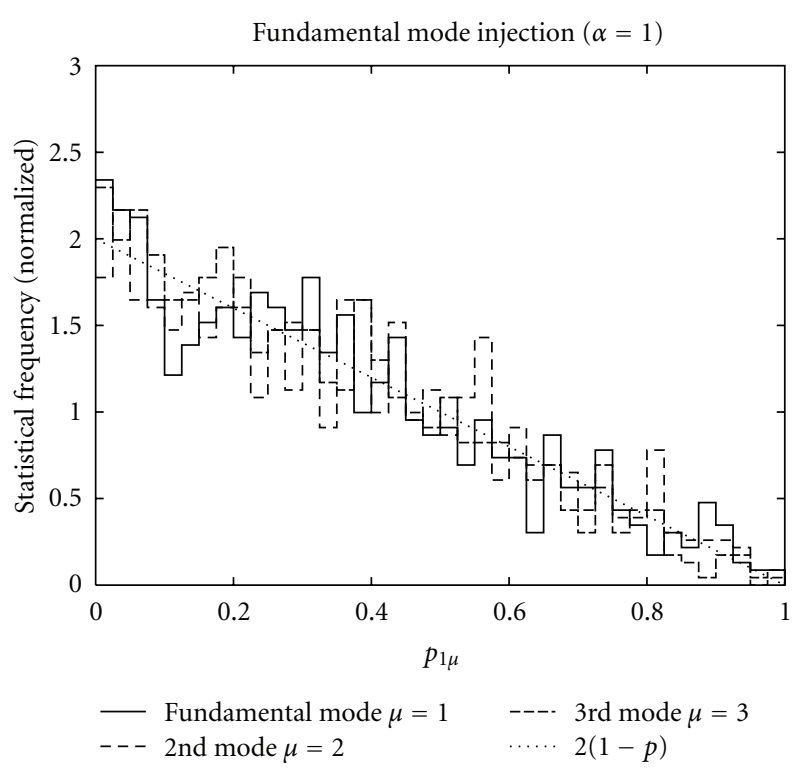

(a)

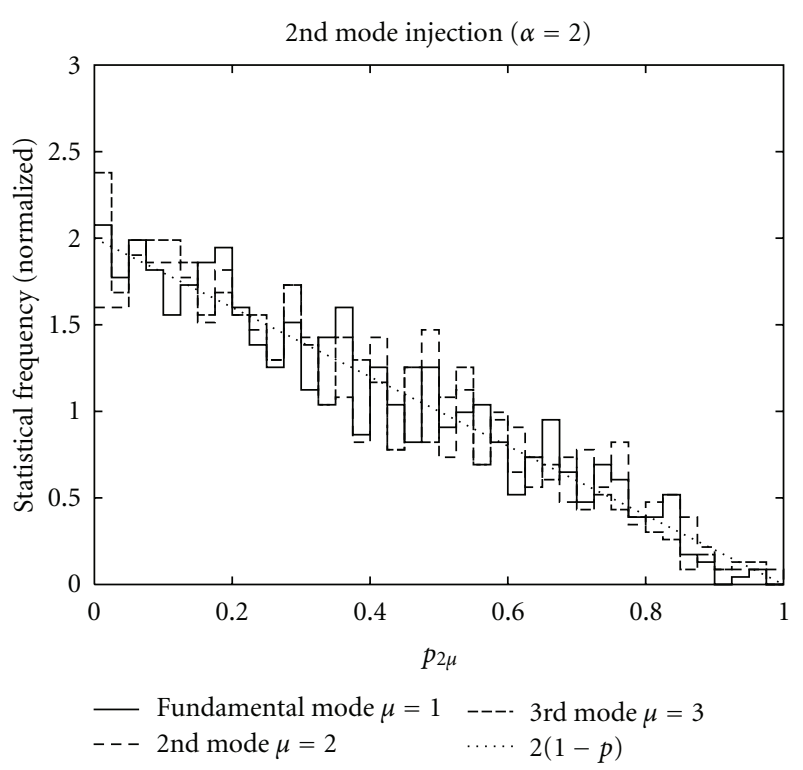

(b)

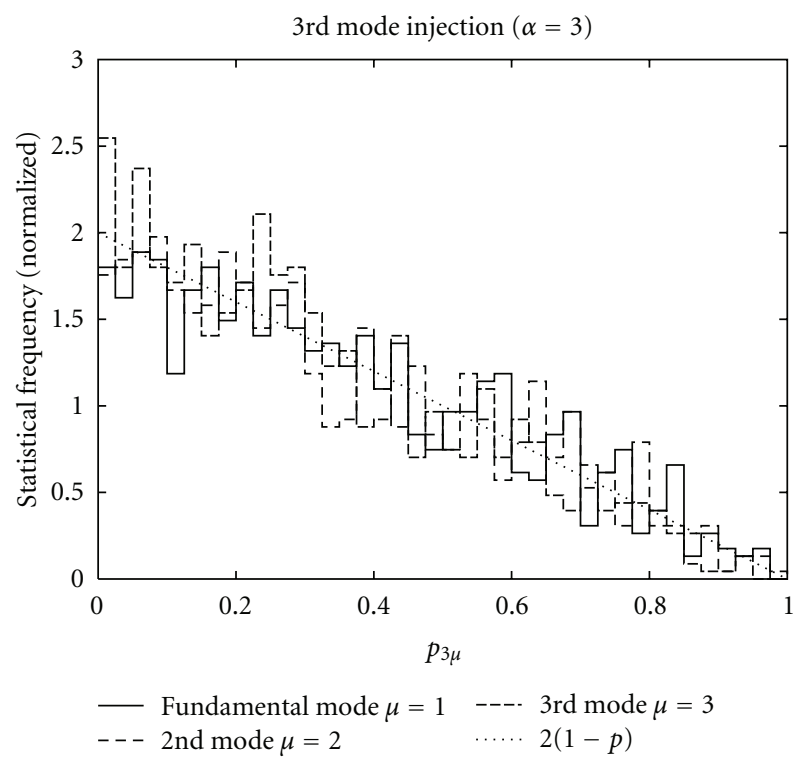

(c)

FIGURE 11: Frequency distributions for the fraction $p_{\alpha \mu}$ of each propagating mode in the transmission current carried by each propagating mode when each of three propagating modes is incident for $K d=\sqrt{32 \pi}, \widetilde{B}=9$.

4.1. Formulation. The $x$ - and $y$-axes are defined in the plane as shown in Figure 1, and the vector potential $\mathbf{A}(\mathbf{r})$ which generates a uniform magnetic field perpendicular to the plane $\mathbf{B}=(0,0,-B)$ is assumed.

The Schrödinger equation of an electron in the presence of a magnetic field is generally written as

$$
\frac{1}{2}[-i \nabla-\mathbf{A}(\mathbf{r})]^{2} \psi(\mathbf{r})=\varepsilon \psi(\mathbf{r})
$$

in a dimensionless form. Here length and energy are scaled by the magnetic length $\ell_{B} \equiv \sqrt{\hbar /|q B|}$ and by the cyclotron energy $\hbar \omega_{c}$, respectively. The cyclotron frequency $\omega_{c}$ is defined by $\omega_{c}=|q B| / m^{*}$ with effective mass of conducting electron $m^{*}$. The vector potential multiplied by the electron charge $q$ is scaled by $\hbar / \ell_{B}$. The scaled total wavenumber is defined by $K \equiv \sqrt{2 \varepsilon}$.

Let us introduce Green's function [30,31]

$$
\begin{gathered}
G\left(\mathbf{r}, \mathbf{r}^{\prime} ; \varepsilon\right)=\exp \left[i \int_{\mathbf{r}^{\prime}}^{\mathbf{r}} \mathbf{A}(\mathbf{s}) \cdot d \mathbf{s}\right] G_{0}(z ; \varepsilon), \\
G_{0}(z ; \varepsilon) \equiv \frac{1}{4 \pi} \Gamma\left(\frac{1}{2}-\varepsilon\right) U\left(\frac{1}{2}-\varepsilon, 1, z\right) e^{-z / 2},
\end{gathered}
$$




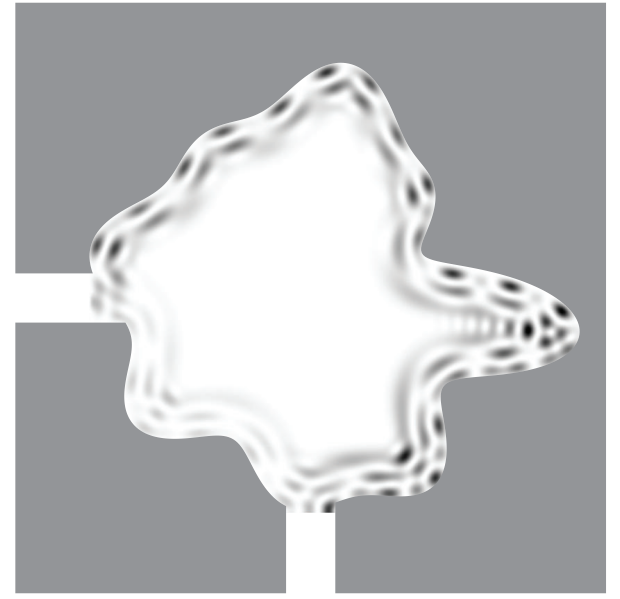

Figure 12: A contour plot of an electron density in a sample dot for $K d=\sqrt{32 \pi}, \widetilde{B}=16$, and the fundamental mode incidence.

which satisfies

$$
\frac{1}{2}\left[i \nabla^{\prime}-\mathbf{A}\left(\mathbf{r}^{\prime}\right)\right]^{2} G\left(\mathbf{r}, \mathbf{r}^{\prime} ; \varepsilon\right)=\varepsilon G\left(\mathbf{r}, \mathbf{r}^{\prime} ; \varepsilon\right)+\delta\left(\mathbf{r}-\mathbf{r}^{\prime}\right),
$$

for any gauge, where $z=\left(\mathbf{r}-\mathbf{r}^{\prime}\right)^{2} / 2$. The function $U(1 / 2-$ $\mathcal{E}, 1, z)$ is a logarithmic solution of the Kummer's equation [32].

Then, the wavefunction within the region enclosed by the boundary $S$ can be expressed as follows in terms of the contour integral along the boundary:

$$
\begin{aligned}
\psi(\mathbf{r})= & \oint\left[G\left(\mathbf{r}, \mathbf{r}^{\prime} ; \varepsilon\right) \nabla^{\prime} \psi\left(\mathbf{r}^{\prime}\right)-\psi\left(\mathbf{r}^{\prime}\right) \nabla^{\prime} G\left(\mathbf{r}, \mathbf{r}^{\prime} ; \varepsilon\right)\right] \cdot \mathbf{n}^{\prime} d S^{\prime} \\
& -2 i \oint G\left(\mathbf{r}, \mathbf{r}^{\prime} ; \varepsilon\right) \psi\left(\mathbf{r}^{\prime}\right) \mathbf{A}\left(\mathbf{r}^{\prime}\right) \cdot \mathbf{n}^{\prime} d S^{\prime}
\end{aligned}
$$

where $\mathbf{n}^{\prime}$ denotes the outward unit normal vector from the region.

When a point $\mathbf{r}$ in the region moves toward the boundary $S$, the following integral equation can be obtained:

$$
\begin{aligned}
c(\mathbf{r}) \psi(\mathbf{r})=\wp \oint d S^{\prime}( & G\left(\mathbf{r}, \mathbf{r}^{\prime}\right) \frac{\partial \psi\left(\mathbf{r}^{\prime}\right)}{\partial n^{\prime}}-\psi\left(\mathbf{r}^{\prime}\right) \frac{\partial G\left(\mathbf{r}, \mathbf{r}^{\prime}\right)}{\partial n^{\prime}} \\
& \left.-2 i G\left(\mathbf{r}, \mathbf{r}^{\prime}\right) \psi\left(\mathbf{r}^{\prime}\right) \mathbf{A}\left(\mathbf{r}^{\prime}\right) \cdot \mathbf{n}^{\prime}\right),
\end{aligned}
$$

where the notations $\wp$ and $\partial / \partial n^{\prime}$ denote the Cauchy principal value integral and the outward normal derivative. The coefficient $c(\mathbf{r})$ is defined by $c(\mathbf{r}) \equiv \theta(\mathbf{r}) / 2 \pi$ when the inner angle formed by the boundary at the point $\mathbf{r}$ on the boundary is $\theta(\mathbf{r})$. If the boundary is smooth at point $\mathbf{r}$, then $c(\mathbf{r})=1 / 2$.

This integral equation is discretized and solved by means of the boundary element method following $[33,34]$.

4.2. Boundary Conditions and Numerical Implementation. It is assumed that the dot is formed by an infinitely high potential. On the boundary, the wavefunction $\psi\left(\mathbf{r}^{\prime}\right)$ is 0 and only the first term on the right-hand side of (10) remains. The boundary of the dot is discretized in steps of $1 / 6$ the incident wavelength. The boundary integral is evaluated in terms of linear interpolation of the normal derivative of the wavefunction. The normal derivative $\partial \psi / \partial n^{\prime}$ of the wavefunction at each node becomes the unknown.

Edge states are formed when electrons are confined in a waveguide subjected to magnetic fields [35]. Although wavefunctions at the orifice should be expanded in terms of the edge states, the influence of magnetic fields will be small when the cyclotron diameter is sufficiently larger than the width of the waveguides. In usual experiments, in addition, the incident waves were injected not through a long waveguide surrounded by rigidly confined potential barrier but through a point contact. Then, electrons will not spend a long enough time to form edge states on passing through the constrictions. The penetration of electrons into the constrictions also prevents electrons from forming edge states. If the width of the waveguide is determined as an effective width of the incident beam, it will be justified to ignore the magnetic field in the emitter. In this paper, further, we are going to show that the statistical transmission probability never depends on the state of the incident wave. So that, in this case, the incident wave may be expressed in terms of any complete sets. Therefore, we put $\mathbf{A}(\mathbf{r})=$ $\left(0, x_{\min }, 0\right)$ within the emitter, that is, in the region of $x<$ $x_{\text {min }}$. It is, then, convenient to employ the Landau gauge $\mathbf{A}=(0, x, 0)$ in the region of $x \geq x_{\min }$ since the vector potential must be continuous even on the $y$-axis.

When an electron wave belonging to the transverse mode $\alpha$ is injected, the total wave function in the emitter of width $d$ is written as

$$
\begin{gathered}
\psi_{\alpha}(\mathbf{r})=e^{-i y x_{\min }}\left(u_{\alpha}^{*}(\mathbf{r})+\sum_{\beta} r_{\alpha \beta} u_{\beta}(\mathbf{r})\right), \\
u_{\alpha}(\mathbf{r}) \equiv \exp \left(-i k_{\alpha} x\right) \sqrt{\frac{2}{d}} \sin \left(\frac{\alpha \pi}{d}\left(y+\frac{d}{2}\right)\right), \\
k_{\alpha} \equiv \sqrt{K^{2}-\left(\frac{\alpha \pi}{d}\right)^{2}}, \quad(\alpha, \beta=1,2, \ldots) .
\end{gathered}
$$

On the orifice of the emitter, the reflection coefficients $r_{\alpha \beta}$ are unknown variables.

Similarly, the wave function at the collector is expanded in terms of the edge states $\chi\left(\mathbf{r}, \kappa_{\mu}\right)[35]$ as

$$
\psi_{\alpha}(\mathbf{r})=\sum_{\mu} t_{\alpha \mu} \chi\left(\mathbf{r}, \kappa_{\mu}\right) .
$$

The edge states, namely, eigenfunctions $\chi\left(\mathbf{r}, \kappa_{\mu}\right)$ are defined by

$$
\chi(\mathbf{r}, \kappa) \equiv \exp (i \kappa y) \varphi(x, \kappa)
$$

where $\varphi(x, \kappa)$ 's are eigenstates of the differential equation

$$
\left[-\frac{d^{2}}{d x^{2}}+(\kappa+x)^{2}\right] \varphi(x, \kappa)=2 \varepsilon \varphi(x, \kappa),
$$




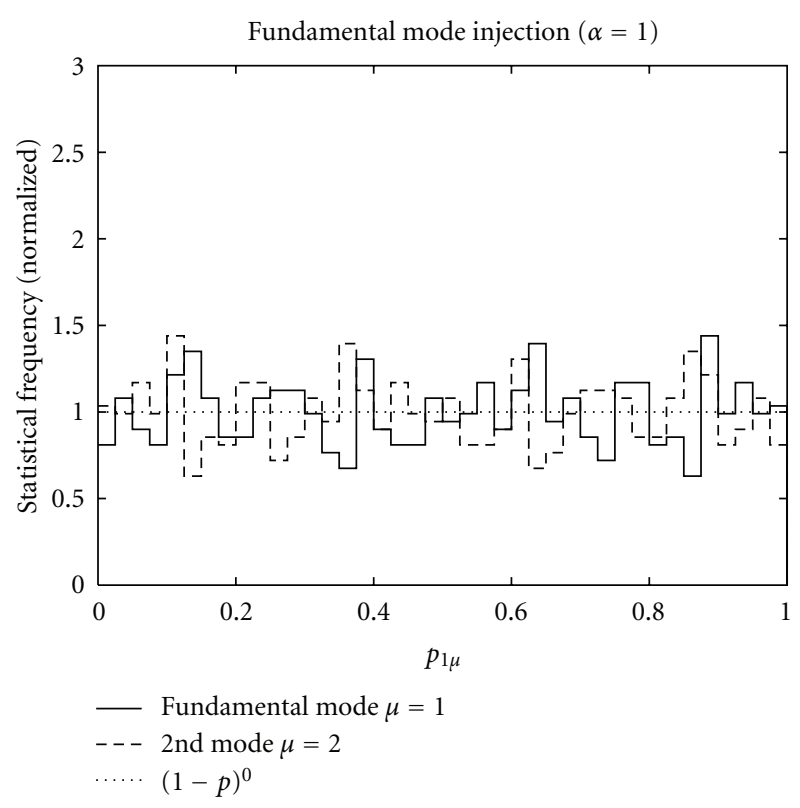

(a)

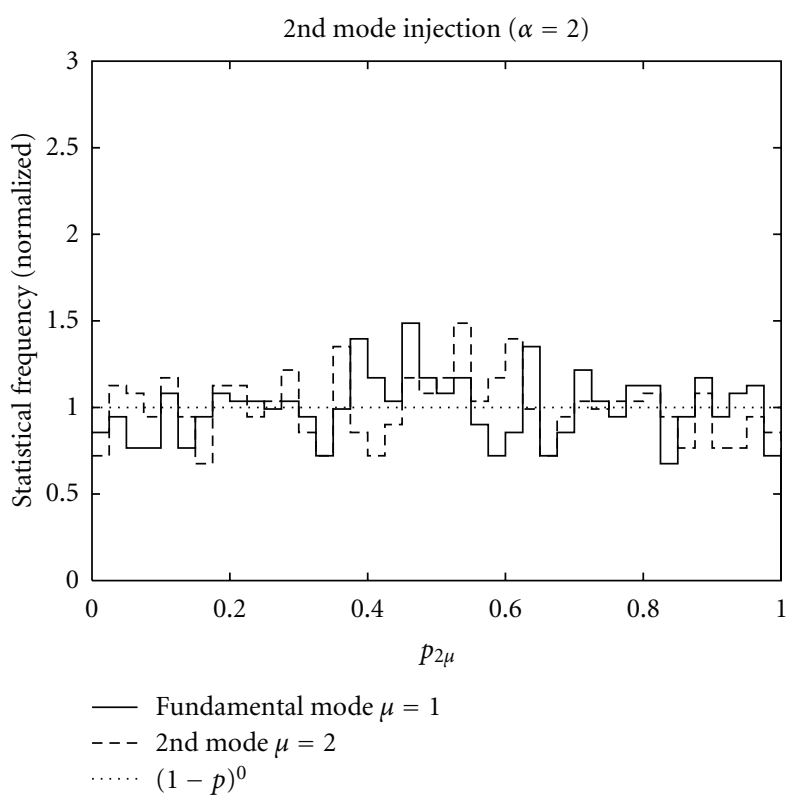

(b)

FIGURE 13: Frequency distributions for the fraction $p_{\alpha \mu}$ of each propagating mode in the transmission current carried by each propagating mode when each of three propagating modes is incident for $K d=\sqrt{32 \pi}, \widetilde{B}=16$.

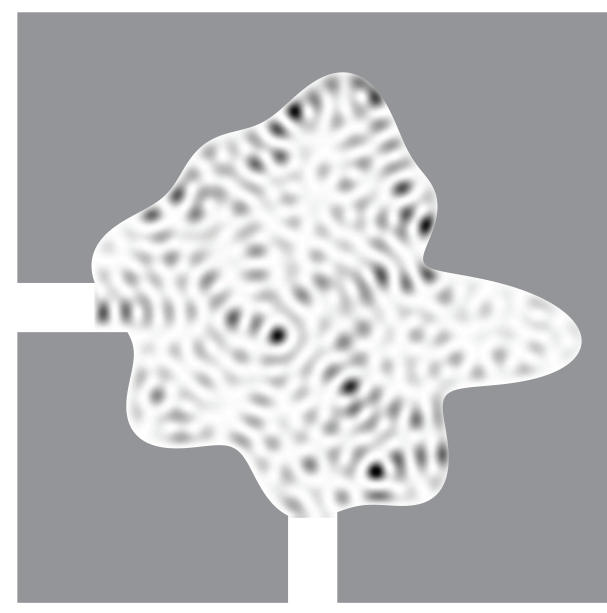

FIGURE 14: A contour plot of an electron density in a sample dot for $K d=\sqrt{20 \pi}, \widetilde{B}=4$, and the fundamental mode incidence.

with boundary conditions $\varphi(x= \pm d / 2, \kappa)=0$ on the walls of the collector. The wavenumber $\kappa_{\mu}$ is selected so that (12) expresses an outgoing wave at the collector. The transmission coefficients $t_{\alpha \mu}$ are unknowns to be determined. Here, note that the eigenwavevector $\kappa$ has complex values for sufficiently large $\varepsilon$ as Schultz et al. pointed out.

The specific discretization procedure for (10) follows the general method using first-order elements. I shall not mention this here since there exist many references which deal with this topic in detail $[33,34]$. The simultaneous equations obtained by discretization are solved for $\partial \psi_{i} / \partial n^{\prime}$, $r_{\alpha \beta}$, and $t_{\alpha \mu}$.
4.3. Evaluation of Physical Quantities. The $\beta$ th propagating mode component of the reflection current at the emitter and the $\mu$ th propagating mode component of the transmission current at the collector are expressed as

$$
\begin{gathered}
j_{\alpha \beta}^{R}=k_{\beta}\left|r_{\alpha \beta}\right|^{2}, \\
j_{\alpha \mu}^{T}=\left|t_{\alpha \mu}\right|^{2} v_{\mu}, \\
v_{\mu} \equiv \int_{-d / 2}^{d / 2}\left(\kappa_{\mu}+x\right)\left|\varphi\left(x, \kappa_{\mu}\right)\right|^{2} d x,
\end{gathered}
$$

respectively. Here, $v_{\mu}$ is the group velocity of the $\mu$ th edge state in the collector. The total reflection current and the total transmission current are given by

$$
j_{\alpha}^{T}=\sum_{\mu} j_{\alpha \mu}^{T},
$$

where the sums over $\beta$ and $\mu$ are taken only over the propagating modes.

When the $\alpha$ th mode is incident from the emitter, the fraction of the transmission current carried by the $\mu$ th propagating mode to the total transmission current within the collector is given by

$$
p_{\alpha \mu} \equiv \frac{j_{\alpha \mu}^{T}}{j_{\alpha}^{T}}
$$

\section{Numerical Results}

In general, once the shape of the dot is specified, the transmission and reflection coefficients are uniquely determined for an incident wave. The fractions of the current 


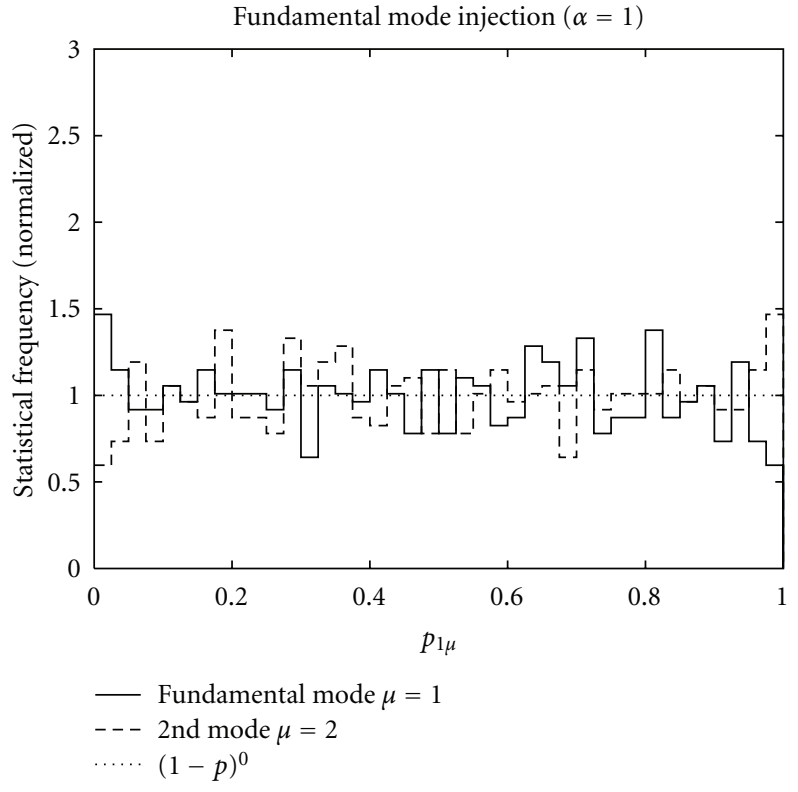

(a)

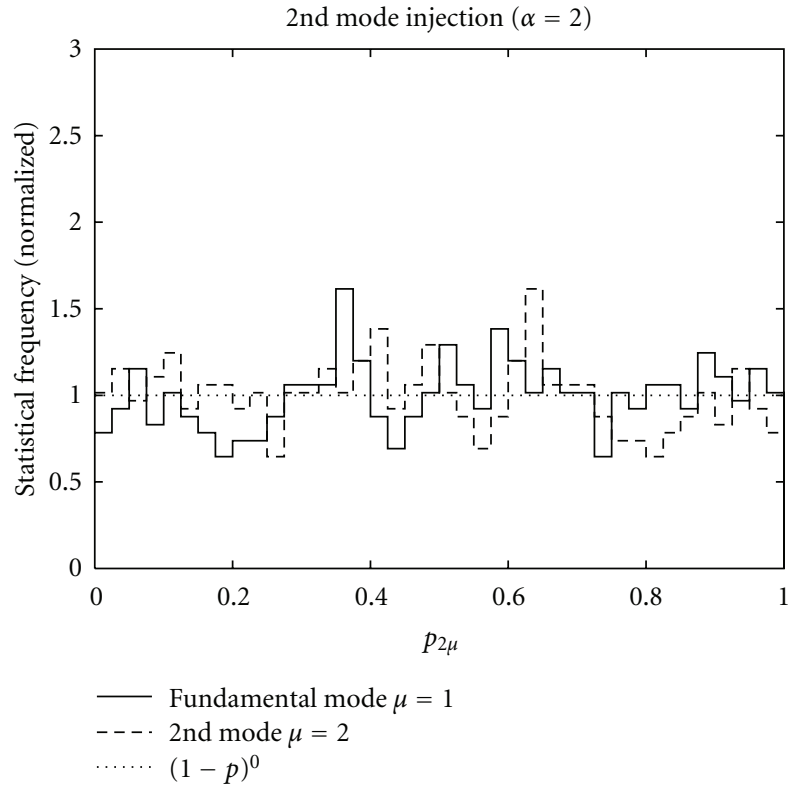

(b)

FIGURE 15: The frequency distribution for the fraction of each propagating mode in the transmission current for the fundamental mode incidence; $K d=\sqrt{20 \pi}, \widetilde{B}=4$.

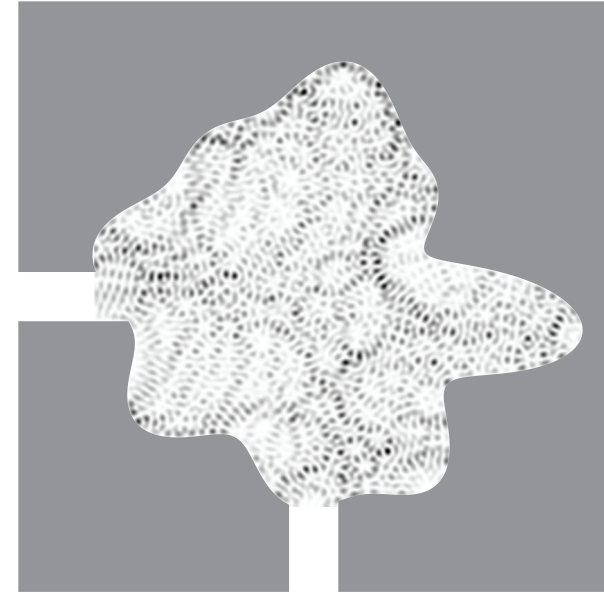

Figure 16: A contour plot of an electron density in a sample dot for $K d=\sqrt{150 \pi}, \widetilde{B}=9$, and the fundamental mode incidence.

carried by each propagating mode in the transmitted and reflected waves depend on the incident mode. As the roughness is increased, however, the wave within the dot is substantially disturbed by scattering in the dot and the statistical characteristics will finally become universal. That has already been confirmed in the absence of a magnetic field.

Hereafter, we employ the dimensionless parameter $\widetilde{B} \equiv$ $B / B_{0}$ for the magnetic field, where $B_{0}$ is defined by $\hbar / q d^{2}$. When the width $d$ of the waveguide is, for example, $250 \mathrm{~nm}$, the magnetic field corresponding to $\widetilde{B}$ is about $0.01 \mathrm{~T}$. The parameter of deformation $a_{\max }$ is set as $a_{\max }=1$, for which an incident wave is sufficiently disturbed and the frequency distribution function of the fraction of the current carried by each propagating mode in the emitter does never depend on the incident mode in the absence of a magnetic field.

At first, we consider the case of $K d=\sqrt{32 \pi}$. In this case, three propagating modes exist in the emitter. Numerical results for $\widetilde{B}=4$ are shown in Figures 8 and 9 . Then, the number of the propagating modes is three, the same as that of for $\widetilde{B}=0$.

Figure 8 is a contour plot of an electron density in a certain sample dot for the fundamental mode incidence. In this figure, we see that the electron wave spreads within the whole dot, and it is disturbed enough, the influence of the magnetic field being not so strong. Figure 9 shows frequency distributions for the fraction of the transmission current carried by each propagating mode when each of the three propagating modes is incident. The abscissa represents $p_{\alpha \mu}$ $(\alpha, \mu=1,2,3)$. These distributions are found to converge to a certain identical distribution. The distribution function never depends on the incident mode, that is universal even in a magnetic field. This fact means that the electron wave is sufficiently disturbed within the dot even in this case. For any incident mode, the probability distribution function for the fraction of the transmission current is $f(p)=2(1-p)$, which is completely in agreement with (1).

Next, results in case of a rather stronger magnetic field $\widetilde{B}=9$ are shown in Figures 10 and 11 .

Even in this case, the number of the propagating modes in the collector is still 3. However, it is found that the cyclotron radius becomes smaller and an electron wave cannot reach around the central part of a dot from the density plot of an electron probability density (Figure 10). In spite of expecting that such change of the quantum state 


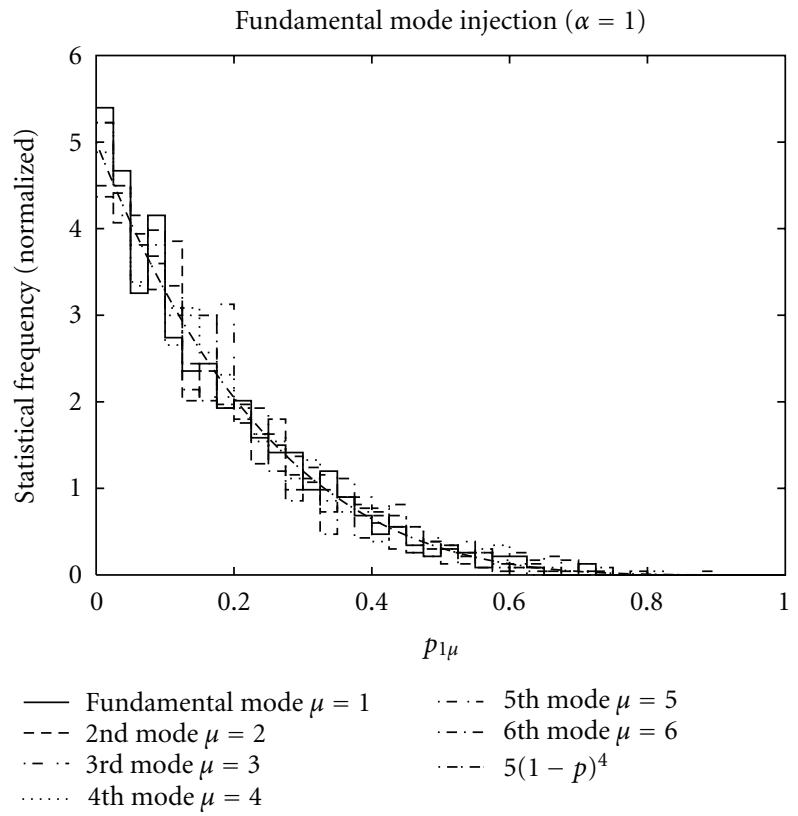

(a)

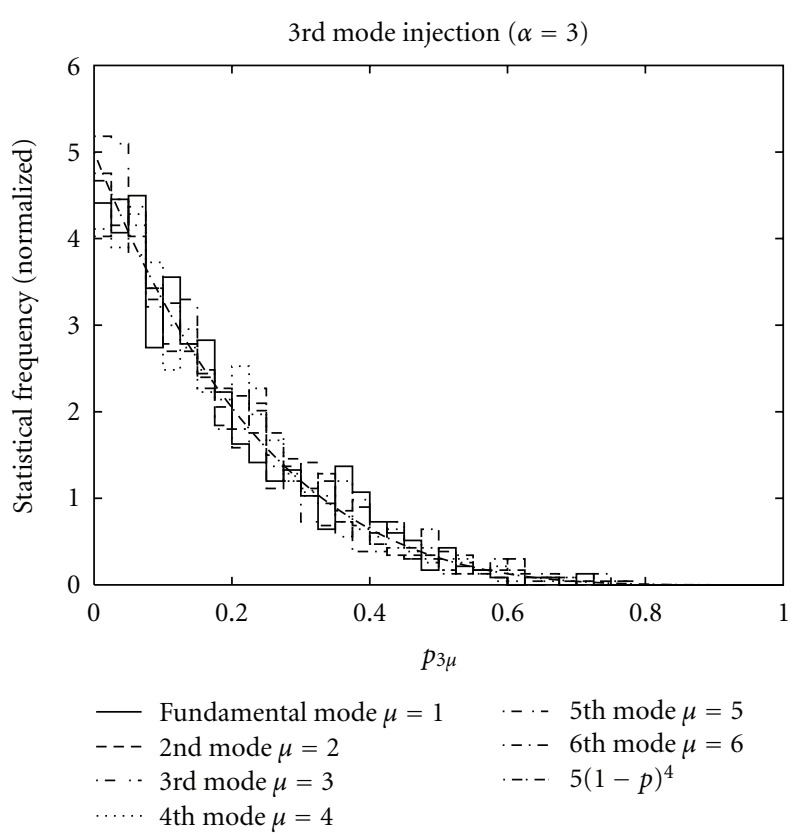

(b)

FIGURE 17: The frequency distribution for the fraction of each propagating mode in the transmission current for the fundamental and third mode incidence, respectively; $K d=\sqrt{150 \pi}, \widetilde{B}=9$.

has big influence on disturbance of an electron wave, the frequency distribution (Figure 11) follows (1) very well.

The results in the case of a further stronger magnetic field are shown in Figures 12 and 13.

Now, the number of the propagating modes in the collector is 2 . The density plot of electron probability density, Figure 12, shows that the electron wave propagates along the wall of the dot forming an edge state. In this situation, the disturbance of an electron wave by scattering on the wall of a randomly deformed dot is not expectable any longer. Nevertheless, the frequency distributions shown in Figure 13 obey (1) for $N=2$.

In order to study variation of $K d$, we show results for $K d=\sqrt{20 \pi}$ and $\widetilde{B}=4$ in Figures 14 and 15. As shown in Figure 14, the electron wave spreads within the whole dot whereas the number of the propagating modes in the collector is 2 , namely, $N=2$. The frequency distribution function shown in Figure 15 obeys (1) for $N=2$ also in this case.

Results for $K d=\sqrt{150 \pi}$ and $\widetilde{B}=4$ are shown in Figures 16 and 17. The wavefunction is distributed all over the dot, and a very fine interference pattern is found in Figure 16. Figure 17 shows that the frequency distribution function satisfies (1) for $N=6$.

The occupation probability refers only to the absolute value of the transmission coefficients, $\left|t_{\alpha \mu}\right|$, not to the phase of them. Now, we pay our attention to phase of $t_{\alpha \mu}$ [36]. In Figure 18, the transmission coefficients $t_{1 \mu}$ of the fundamental, 2nd, and $3 \mathrm{rd}$ modes for $K d=\sqrt{32 \pi}, \widetilde{B}=9$ and fundamental mode incidence are plotted for all samples of shape in the complex plane. We find that sample points for any mode are isotropically distributed around the origin. This fact implies that the phase of $t_{\alpha \mu}$ are random.

We also see that the distribution spreads as the mode number. In fact, the variance in the radial direction of each distribution is inverse to the group velocity $v_{\mu}$, so that

$$
\left\langle\left|t_{\alpha \mu}\right|^{2}\right\rangle_{\text {shape }} \propto \frac{1}{v_{\mu}},
$$

with the accuracy better than $1 \%$. This reflects that the mean value $\left\langle j_{\alpha \mu}^{T}\right\rangle_{\text {shape }}$ of the current of each propagating mode $\mu$ is independent of the belonging mode $\mu$. This is consistent with (23).

\section{Discussions and Conclusions}

By means of a classical model, that is, quantized billiard model, it is shown that the statistical properties of the transmission current out of a randomly deformed quantum dot are equivalent to the properties of infinitely large reservoir model. Thus, in the present paper, I have proposed this model as a model of an electron reservoir in quantum mechanics.

On the basis of the reservoir model, the statistical properties of the transmission current are quantum-mechanically computed. Then, the frequency distribution for the fraction of the transmission current carried by each propagating mode in a collector obeys (1) for any wavenumber $K d$ and any magnetic field $\widetilde{B}$. This rule is namely universal.

Here, let us consider physical explanation of this rule. Suppose, for example, that three propagating modes exist in 


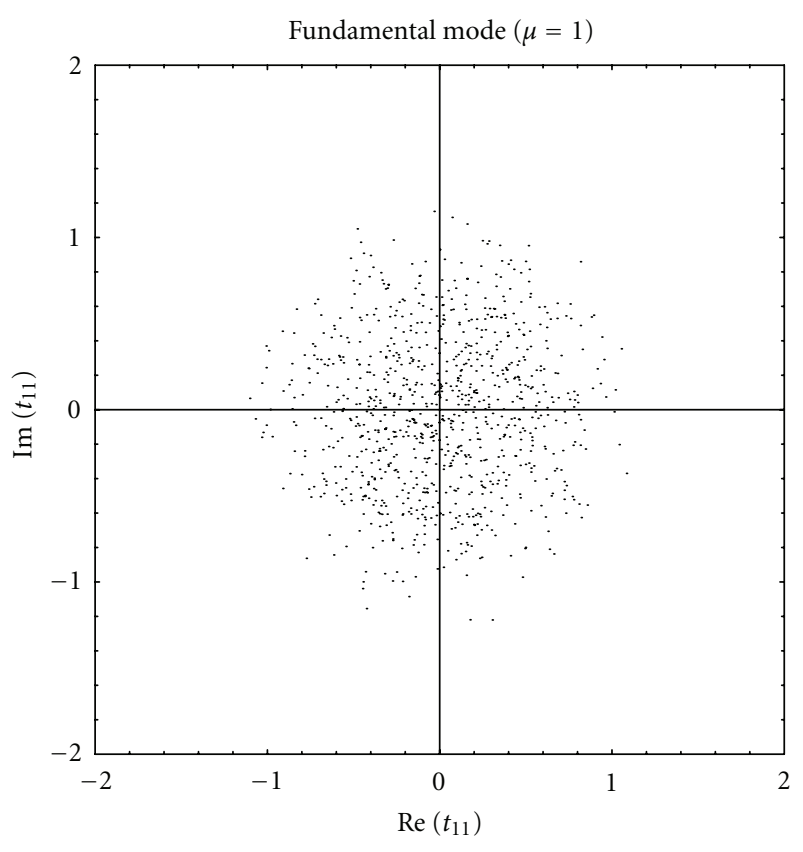

(a)

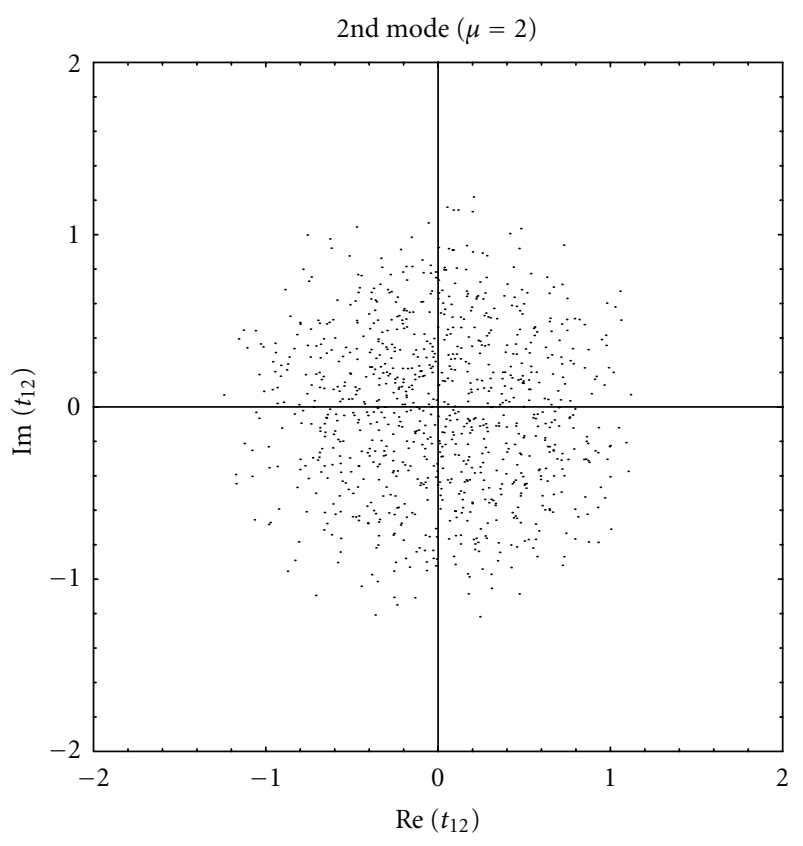

(b)

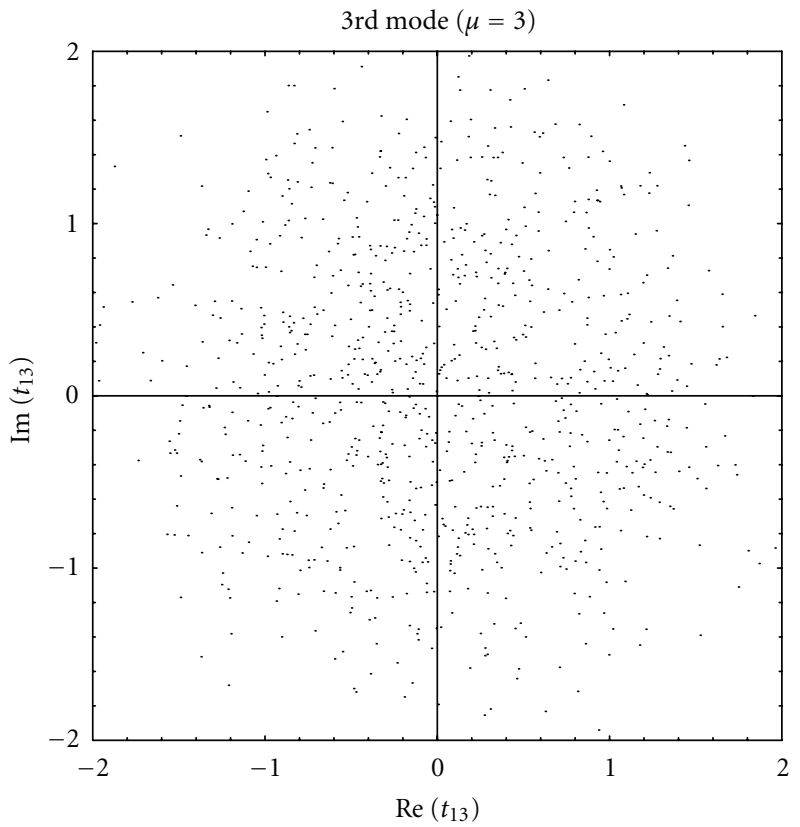

(c)

Figure 18: Plots of transmission coefficients $t_{1 \mu}$ of the fundamental, 2nd, and 3rd modes for all samples of shape in the complex plane. The incident wave belongs to the fundamental mode; $K d=\sqrt{32 \pi}$ and $\widetilde{B}=9$.

the collector. The reason of this distribution function can be interpreted as follows. Let $p_{1}, p_{2}$, and $p_{3}$ be the fractions of the current carried by the fundamental, the second, and the third mode, respectively. A certain sample is represented by one point on the first quadrant of a plane

$$
\sum_{\mu=1}^{3} p_{\mu}=1, \quad 0 \leq p_{\mu} \leq 1,(\mu=1,2,3),
$$

in the three-dimensional space spanned by the $p_{1}, p_{2}$ and $p_{3}$ axes (see Figure 19).
If the point $\left(p_{1}, p_{2}, p_{3}\right)$ is randomly (uniformly) distributed within the equilateral triangle equation (21), then the frequency $f(p)$ with the value of one axis such as $p_{1}$ fixed at $p_{1}=p$ is proportional to the length of the line segment formed by the intersection of plane $p_{1}=p$ and the equilateral triangle. Hence, $f(p)$ is proportional to $(1-p)$. Substituting it into the normalization condition

$$
\int_{0}^{1} f(p) d p=1
$$




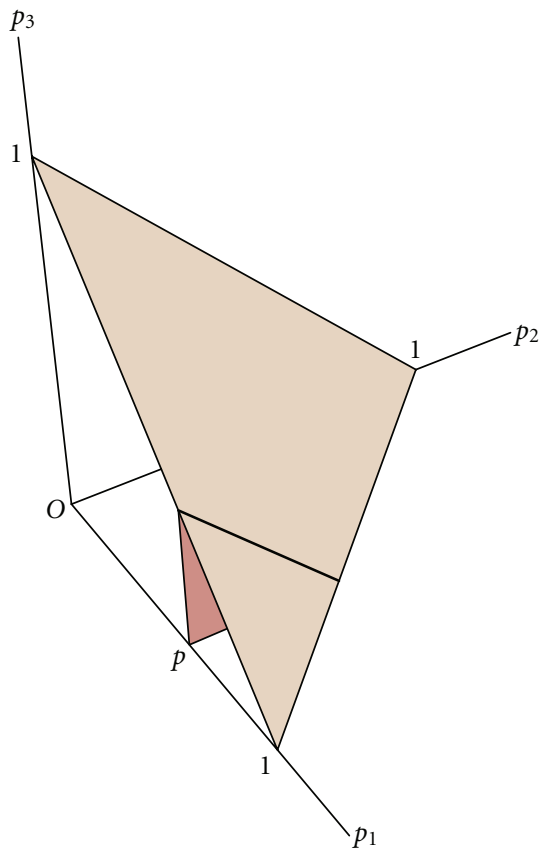

FIGURE 19: The first quadrant of plane equation (21).

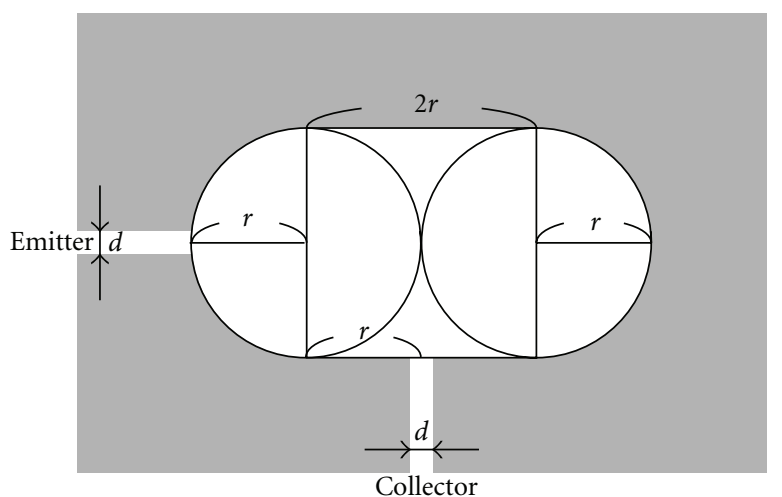

Figure 20: A stadium-shaped quantum dot with an emitter and a collector.

we obtain $f(p)=2(1-p)$.

The fact that the frequency distribution function has this form reveals that the transmission current is randomly distributed over all propagation modes. In addition, we have

$$
\int_{0}^{1} p f(p) d p=\frac{1}{3}
$$

so that all modes carry equal currents on average.

If the current is assumed to be distributed randomly over the propagating modes when $N$ propagating modes exist, the distribution function can be derived by extending the considerations for the case of three propagating modes to $\mathrm{N}$-dimensional space [37]. The normalized distribution function is obtained as

$$
f(p)=(N-1)(1-p)^{N-2}, \quad(N \geq 1) .
$$

Then, the average and the variance of $p$ are given by

$$
\begin{gathered}
\langle p\rangle=\int_{0}^{1} p f(p) d p=\int_{0}^{1} p(N-1)(1-p)^{N-2} d p=\frac{1}{N}, \\
\left\langle p^{2}\right\rangle-\langle p\rangle^{2}=\frac{2}{N(N+1)}-\left(\frac{1}{N}\right)^{2}=\frac{1}{N^{2}} \frac{N-1}{N+1},
\end{gathered}
$$

respectively. The variance is 0 for $N=1$, it takes the maximum value, $1 / 12$ for $N=2$ and for $N \geq 2$, it decreases in monotone and approaches 0 as $N$ becomes large. The ratio of the standard deviation and the average, however, is given by

$$
\frac{\sqrt{\left\langle p^{2}\right\rangle-\langle p\rangle^{2}}}{\langle p\rangle}=\sqrt{\frac{N-1}{N+1}},
$$

it increases monotonously with $N$, and approaches 1 on the limit of $N \rightarrow \infty$.

For $N=1,(24)$ becomes $f(p)=0$ for $p \neq 1$, and $f(p)$ diverges for $p=1$. Further, $f(p)$ satisfies (22) and is certainly $f(p)=\delta(p-1)$. Namely, only one propagating mode carries all of the current.

Thus, this distribution function reflects the law of equipartition of current.

The key point of the model of a reservoir is sufficient disturbance of the electron wave within a quantum dot. Not a few researchers often expect that an electron wave is enough dephased within a chaos quantum dot and that it can become a model of a reservoir.

I have already confirmed the transport properties of a stadium-shaped dot with an emitter and a collector as an example of a chaotic dot, quantum mechanically and classically [18-21]. Both cases in the absence and the presence of a magnetic field have been analyzed whereas I restrict myself to showing only the results of quantummechanical analysis for zero magnetic field.

Now, we consider a stadium-shaped quantum dot shown in Figure 20.

The wavenumber dependence of the fraction of the current $p_{\alpha \mu}$ carried by each propagating mode $\mu$ in the collector is shown in Figure 21 for cases that each propagating mode $\alpha$ in the emitter is injected. Here, the size of the dot is set as $r / d=10$. From Figure 21, we see that the fraction of the current carried by each propagating mode strongly depends on the incident mode and changes rapidly to the wavenumber.

Figure 22 shows the size $r / d$ dependence of the fraction of the current $p_{\alpha \mu}$ carried by each propagating mode $\mu$ in the collector for each incident mode $\alpha$. The wavenumber $K d$ is set to 10. It is shown that the fraction of the current carried by each propagating mode strongly is also dependent on the size of the dot.

The electron density for $K d=7, r / d=10$, and the fundamental mode incidence is plotted in Figure 23. We see that in this case an eigen mode of high symmetry is excited in the dot. When the wavenumber or the size of a dot changes, space variation of a wavefunction of an excited eigen mode 


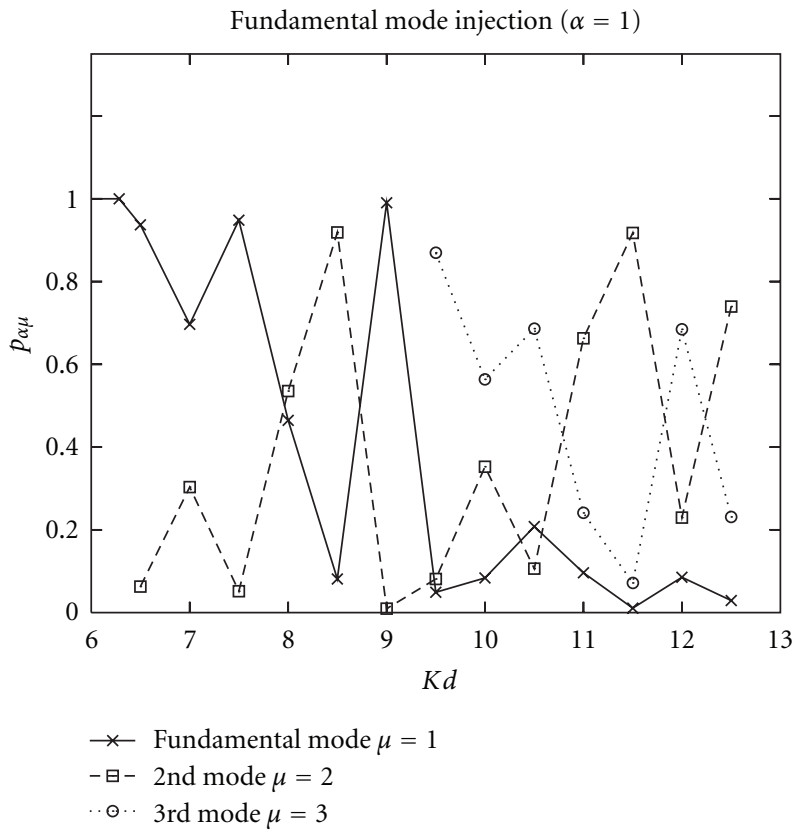

(a)

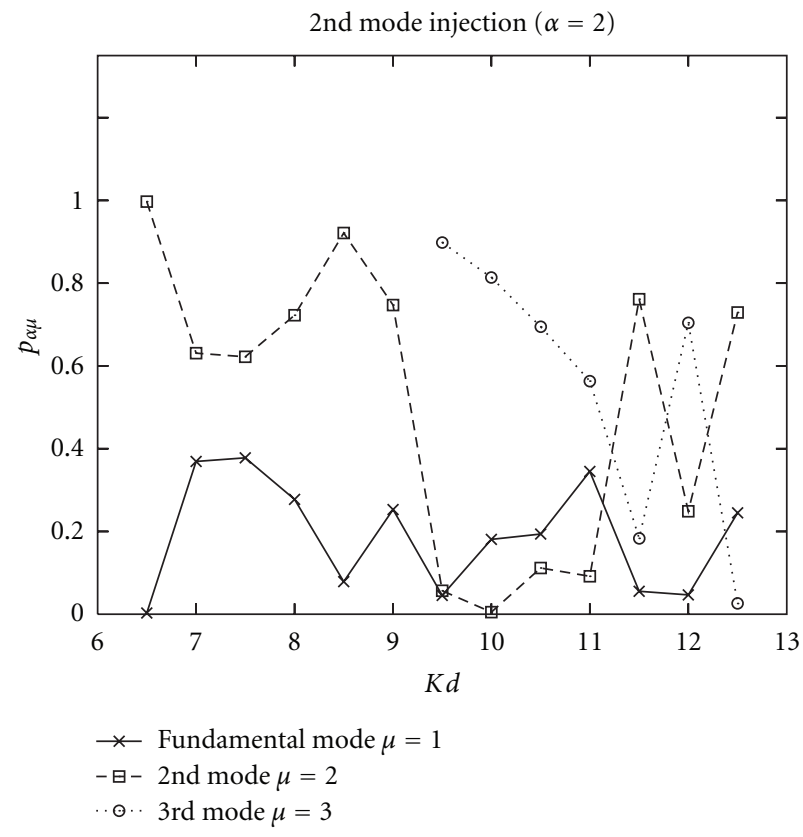

(b)

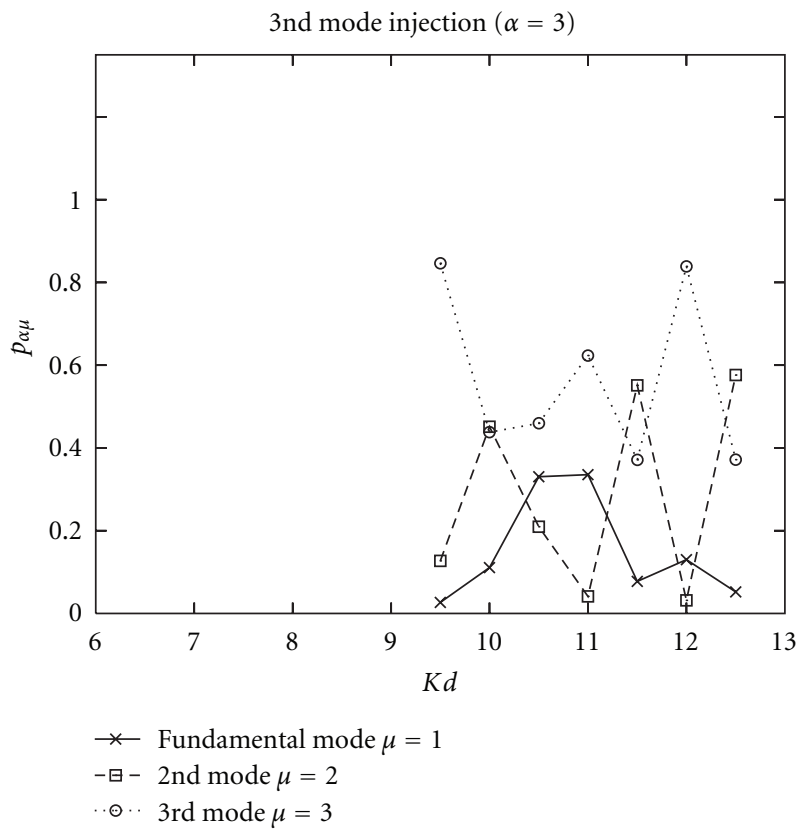

(c)

FIGURE 21: Wavenumber dependence of the fraction $p_{\alpha \mu}$ in the transmission current carried by each propagating mode $\mu$ when each propagating mode $\alpha$ is injected. The size of the dot is set as $r / d=10$. 


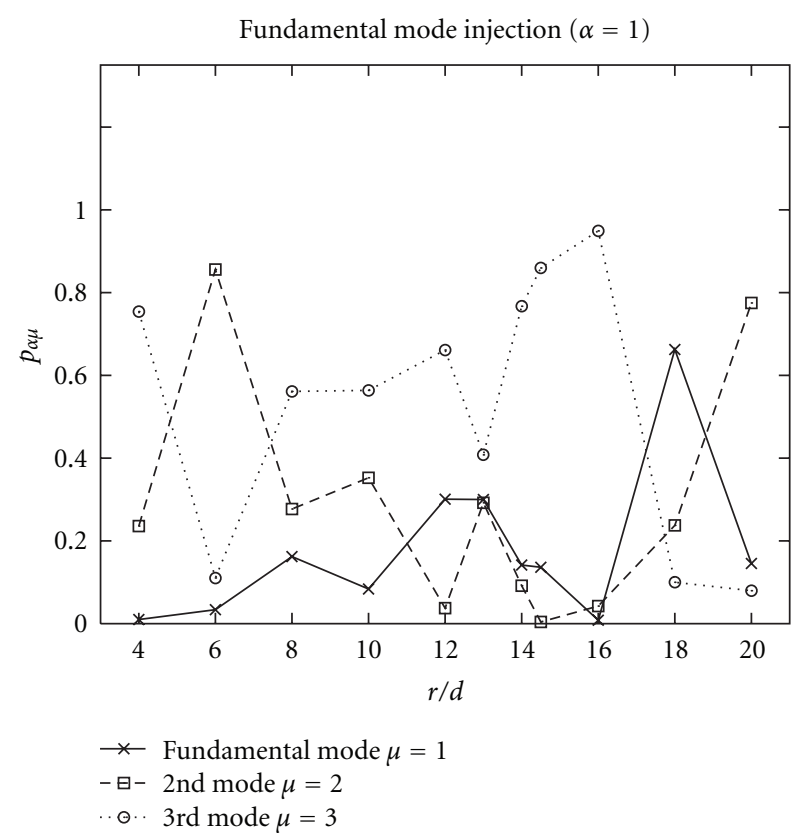

(a)

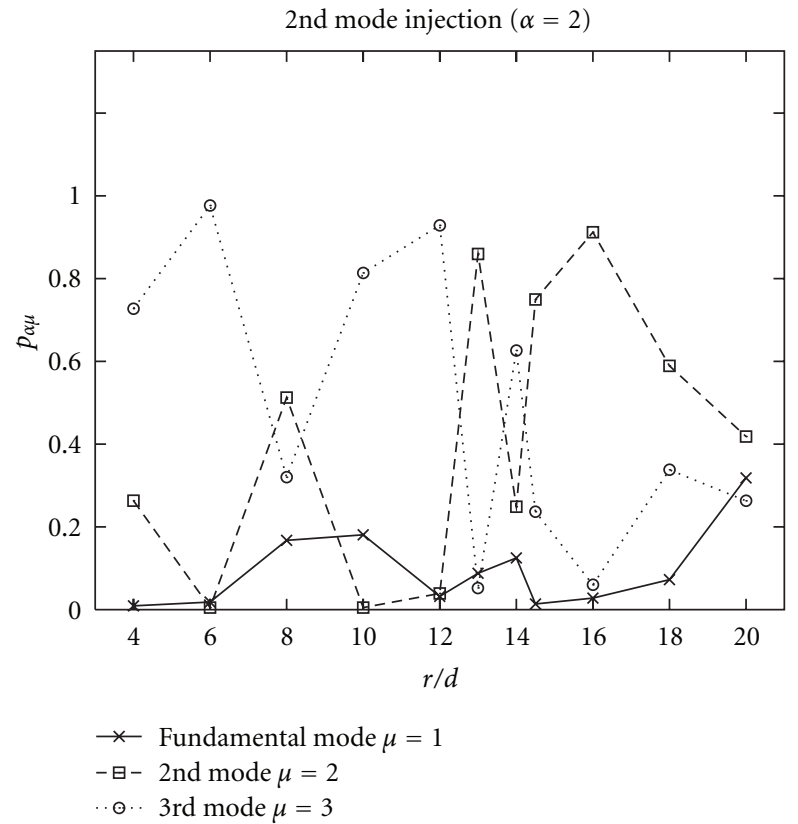

(b)

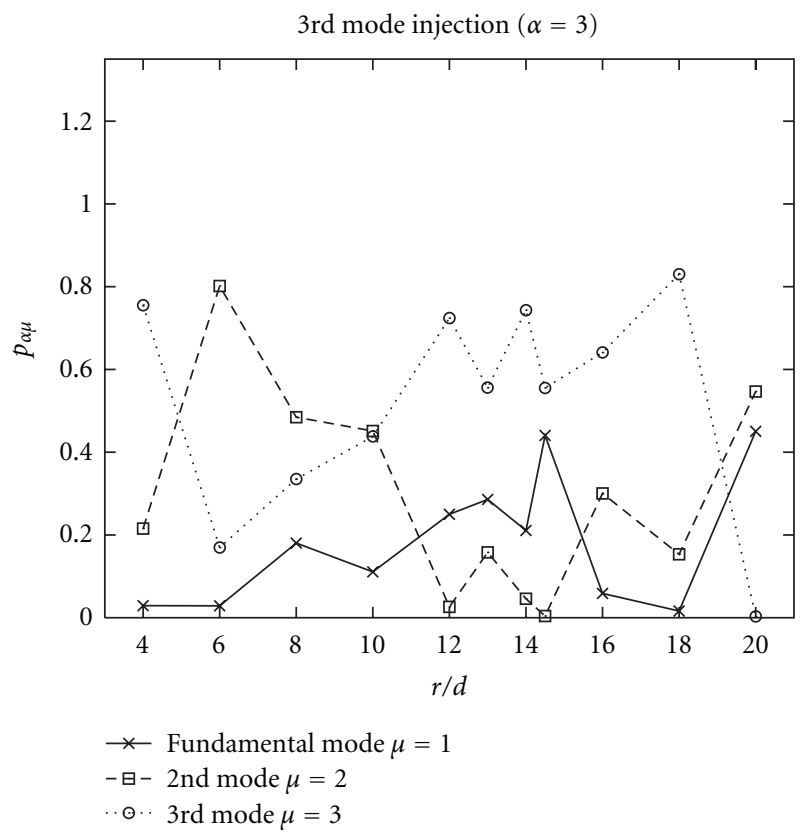

(c)

FIgURE 22: Size $r / d$ dependence of the fraction $p_{\alpha \mu}$ in the transmission current carried by each propagating mode $\mu$ when each propagating mode $\alpha$ is injected. The wavenumber is set as $K d=10$. 


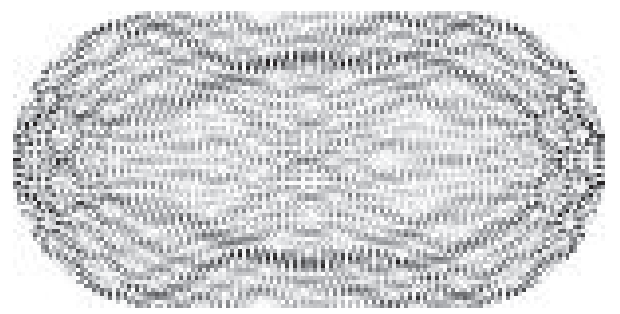

FIgURE 23: A contour plot of an electron density for $K d=7, r / d=$ 10 , and the fundamental mode incidence.

also changes. Then, coupling of a wave function in a collector with that in a dot also changes. Therefore, universal transport property is never realized at least in a finite dot.

Even if we interchange the emitter with the collector or introduce a magnetic field, the tendency of the property does not change at all. Thus, it is confirmed that a finite size dot cannot be a model of a reservoir even if it is chaotic.

\section{References}

[1] C. W. J. Beenakker and H. Van Houten, "Billiard model of a ballistic multiprobe conductor," Physical Review Letters, vol. 63, no. 17, pp. 1857-1860, 1989.

[2] K. Kawamura, T. Ueta, and H. Sawano, "Fraunhoffer diffraction of electrons in anisotropic media," Japanese Journal of Applied Physics, Part 1, vol. 31, no. 2, pp. 317-326, 1992.

[3] M. Saito, M. Takatsu, M. Okada, and N. Yokoyama, "Analysis of the angular distribution of electrons injected through a quantum point contact by use of a Greens function with a weak-magnetic-field approximation," Physical Review B, vol. 46, no. 20, pp. 13220-13233, 1992.

[4] T. Ueta, "Statistical properties of quantum transport through two-dimensional random-shaped quantum dots," Electronics and Communications in Japan, Part II, vol. 83, no. 9, pp. 4248, 2000.

[5] T. Ueta, "Analysis of statistical transport properties of twodimensional random-shaped quantum dots by means of the quantized billiard model," Denshi Joho Tsushin Gakkai Ronbunshi, vol. 83, pp. 359-631, 2000 (Japanese).

[6] H. Bruus and A. D. Stone, "Quantum chaos in a deformable billiard: applications to quantum dots," Physical Review B, vol. 50, no. 24, pp. 18275-18287, 1994.

[7] H. U. Baranger and P. A. Mello, "Mesoscopic transport through chaotic cavities: a random S-matrix theory approach," Physical Review Letters, vol. 73, no. 1, pp. 142-145, 1994.

[8] K. M. Frahm and D. L. Shepelyansky, "Quantum localization in rough billiards," Physical Review Letters, vol. 78, no. 8, pp. 1440-1443, 1997.

[9] M. Büttiker, "Scattering theory of current and intensity noise correlations in conductors and wave guides," Physical Review B, vol. 46, no. 19, pp. 12485-12507, 1992.

[10] R. A. Jalabert, J.-L. Pichard, and C. W. J. Beenakker, "Universal quantum signatures of chaos in ballistic transport," Europhysics Letters, vol. 27, pp. 255-260, 1994.

[11] X. Yang, H. Ishio, and J. Burgdörfer, "Statistics of magnetoconductance in ballistic cavities," Physical Review B, vol. 52, no. 11, pp. 8219-8225, 1995.

[12] J. P. Bird, K. Ishibashi, D. K. Ferry, Y. Ochiai, Y. Aoyagi, and T. Sugano, "Spectral characteristics of conductance fluctuations in ballistic quantum dots: the influence of finite magnetic field and temperature," Physical Review B, vol. 52, no. 11, pp. 82958304, 1995.

[13] K.-F. Berggren, Z.-L. Ji, and T. Lundberg, "Origin of conductance fluctuations in large circular quantum dots," Physical Review B, vol. 54, no. 16, pp. 11612-11621, 1996.

[14] R. Akis, D. K. Ferry, and J. P. Bird, "Magnetotransport fluctuations in regular semiconductor ballistic quantum dots," Physical Review B, vol. 54, no. 24, pp. 17705-17715, 1996.

[15] I. V. Zozoulenko, R. Schuster, K.-F. Berggren, and K. Ensslin, "Ballistic electrons in an open square geometry: selective probing of resonant-energy states," Physical Review B, vol. 55, no. 16, pp. R10209-R10212, 1997.

[16] I. V. Zozoulenko and K. F. Berggren, "Quantum scattering, resonant states, and conductance fluctuations in an open square electron billiard," Physical Review B, vol. 56, no. 11, pp. 6931-6941, 1997.

[17] T. Blomquist and I. V. Zozoulenko, "Magnetoconductance fluctuations and weak localization in quantum dots: reliability of the semiclassical approach," Physical Review B, vol. 64, no. 19, Article ID 195301, 2001.

[18] K. Nakamura and H. Ishio, "Quantum transport in open billiards: comparison between circle and stadium," Journal of the Physical Society of Japan, vol. 61, no. 11, pp. 3939-3944, 1992.

[19] Y. Wang, J. Wang, and H. Guo, "Magnetoconductance of a stadium-shaped quantum dot: a finite-element-method approach," Physical Review B, vol. 49, no. 3, pp. 1928-1934, 1994.

[20] K. Nakamura, K. Ito, and Y. Takane, "Magnetoconductance in open stadium billiard: quantum analogue of transition from chaos to tori," Journal of the Physical Society of Japan, vol. 63, no. 9, pp. 3210-3213, 1994.

[21] K. Nakamura, K. Ito, and Y. Takane, "Magneto-conductance in open billiards: comparison between circle and stadium," Journal of Physics A, vol. 27, no. 17, article 021, pp. 5889-5896, 1994.

[22] J. P. Bird, D. M. Olatona, R. Newbury et al., "Lead-induced transition to chaos in ballistic mesoscopic billiards," Physical Review B, vol. 52, no. 20, pp. R14336-R14339, 1995.

[23] C. D. Schwieters, J. A. Alford, and J. B. Delos, "Semiclassical scattering in a circular semiconductor microstructure," Physical Review B, vol. 54, no. 15, pp. 10652-10668, 1996.

[24] Y. Takane and K. Nakamura, "Influence of small-angle diffraction on the ballistic conductance fluctuations in chaotic billiards," Journal of the Physical Society of Japan, vol. 67, no. 2, pp. 397-400, 1998.

[25] J. P. Bird, R. Akis, D. K. Ferry et al., "Lead-orientationdependent wave function scarring in open quantum dots," Physical Review Letters, vol. 82, no. 23, pp. 4691-4694, 1999.

[26] H. Ishio, "Resonance poles and width distribution for timereversal transport through mesoscopic open billiards," Physical Review E, vol. 62, no. 3, pp. R3035-R3038, 2000.

[27] S. Oberholzer, E. V. Sukhorukov, and C. Schönenberger, "Crossover between classical and quantum shot noise in chaotic cavities," Nature, vol. 415, no. 6873, pp. 765-767, 2002.

[28] M. Planck, "Ueber das gesetz der energieverteilung im normalspectrum," Annalen der Physik, vol. 309, pp. 553-563, 1901.

[29] T. Ueta, "Quantum-mechanical study of electron focusing spectrum and quantized billiard model," Journal of the Physical Society of Japan, vol. 64, no. 12, pp. 4813-4823, 1995. 
[30] T. Ueta, "Green's function of a charged particle in magnetic fields," Journal of the Physical Society of Japan, vol. 61, no. 12, pp. 4314-4324, 1992.

[31] V. V. Dodonov, I. A. Malkin, and V. I. Man'ko, “The green function of the stationary schrödinger equation for a particle in a uniform magnetic field," Physics Letters A, vol. 51, no. 3, pp. 133-134, 1975.

[32] M. Abramowitz and I. A. Stegun, Handbook of Mathematical Functions, Dover, New York, NY, USA, 1970.

[33] T. Ueta, "Boundary element method for electron waves in uniform magnetic fields," Engineering Analysis with Boundary Elements, vol. 17, no. 1, pp. 69-74, 1996.

[34] C. A. Brebbia, The Boundary Element Method for Engineers, Pentech Press, London, UK, 1978.

[35] R. L. Schult, H. W. Wyld, and D. G. Ravenhall, "Quantum Hall effect and general narrow-wire circuits," Physical Review B, vol. 41, no. 18, pp. 12760-12780, 1990.

[36] T. Ueta, "Statistical properties of phases of transmission and reflection coefficients of two-dimensional random-shaped quantum dots," Denshi Joho Tsushin Gakkai Ronbunshi, vol. 82, pp. 653-655, 1999 (Japanese).

[37] D. E. Barton and F. N. David, "Some notes on ordered random intervals," Journal of the Royal Statistical Society, vol. 18, pp. 79-94, 1956. 

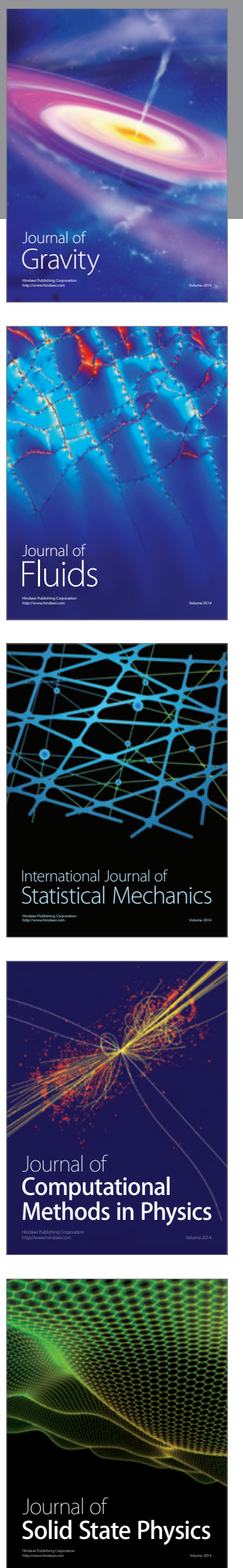

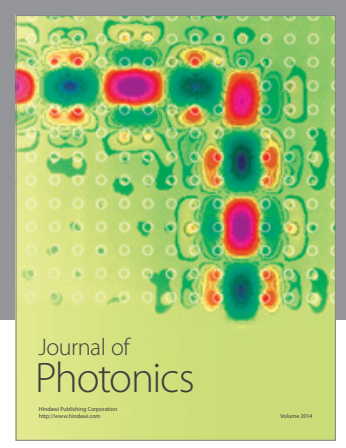

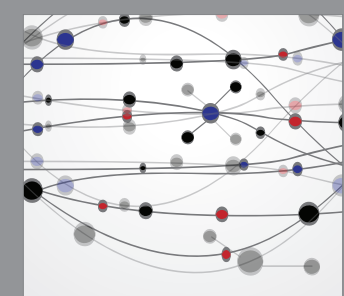

The Scientific World Journal
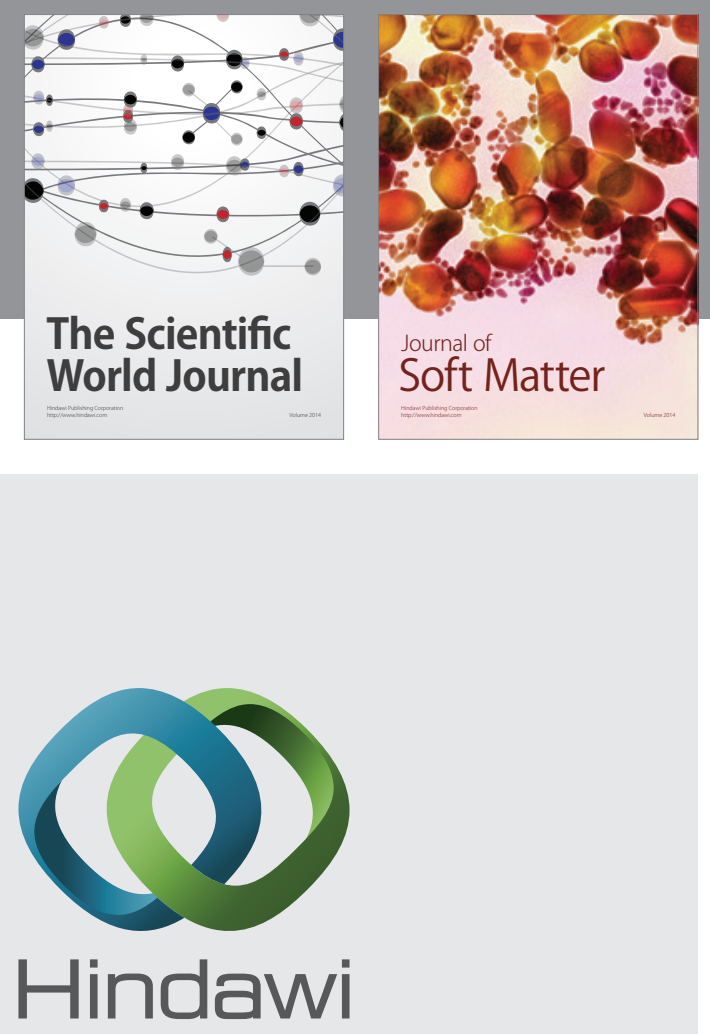

Submit your manuscripts at

http://www.hindawi.com
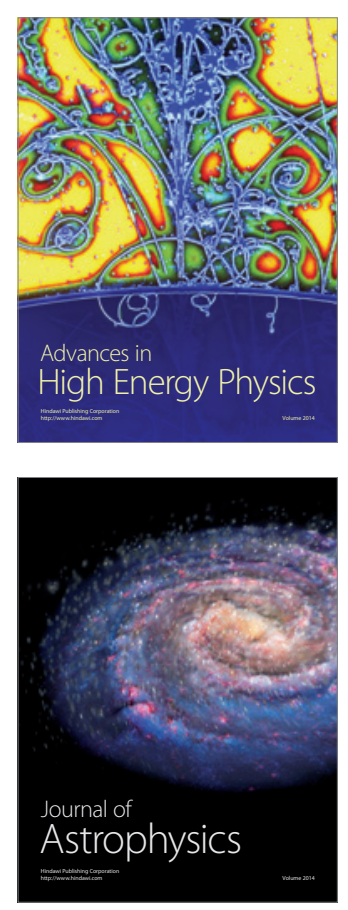
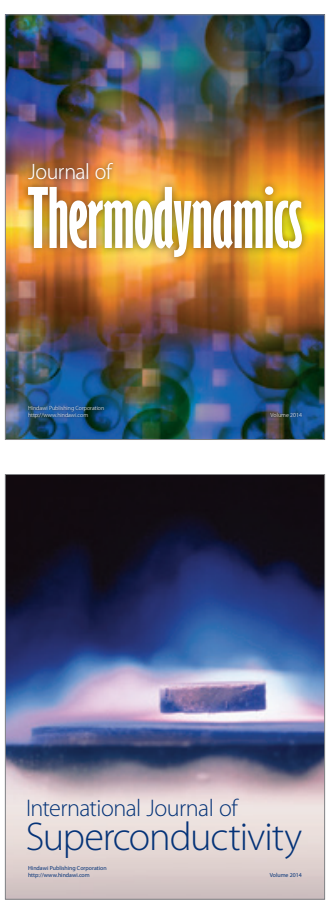
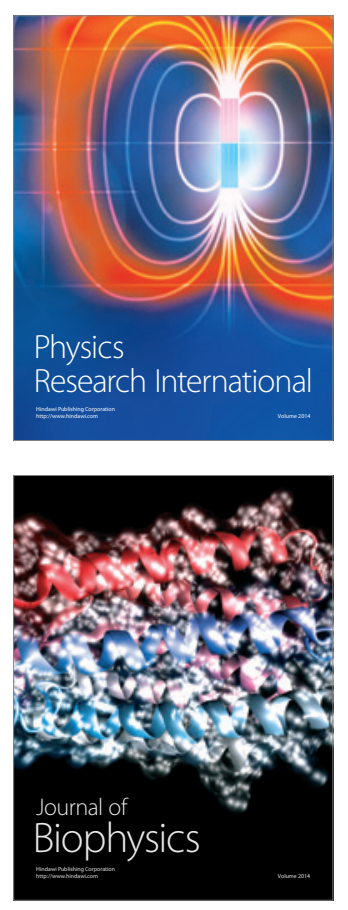
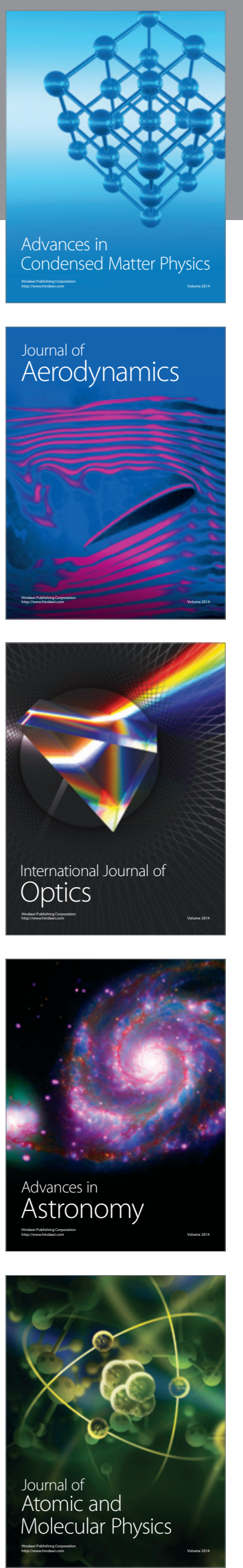